\title{
Bodily action penetrates affective perception
}

Carlo Fantoni, Sara Rigutti, Walter Gerbino

Fantoni and Gerbino (2014) showed that subtle postural shifts associated with reaching can have a strong hedonic impact and affect how actors experience facial expressions of emotion. Using a novel Motor Action Mood Induction Procedure (MAMIP), they found consistent congruency effects in participants who performed a facial emotion identification task after a sequence of visually-guided reaches: a face perceived as neutral in a baseline condition appeared slightly happy after comfortable actions and slightly angry after uncomfortable actions. However, skeptics about the penetrability of perception (Zeimbekis \& Raftopoulos, 2015) would consider such evidence insufficient to demonstrate that observer's internal states induced by action comfort/discomfort affect perception in a top-down fashion. The action-modulated mood might have produced a back-end memory effect capable of affecting post-perceptual and decision processing, but not front-end perception.

Here, we present evidence that performing a facial emotion detection (not identification) task after MAMIP exhibits systematic mood-congruent sensitivity changes, rather than response bias changes attributable to cognitive set shifts; i.e., we show that observer's internal states induced by bodily action can modulate affective perception. The detection threshold for happiness was lower after fifty comfortable than uncomfortable reaches; while the detection threshold for anger was lower after fifty uncomfortable than comfortable reaches. Action valence induced an overall sensitivity improvement in detecting subtle variations of congruent facial expressions (happiness after positive comfortable actions, anger after negative uncomfortable actions), in the absence of significant response bias shifts. Notably, both comfortable and uncomfortable reaches impact sensitivity in an approximately symmetric way relative to a baseline inaction condition. All of these constitute compelling evidence of a genuine top-down effect on perception: specifically, facial expressions of emotion are penetrable by action-induced mood. Affective priming by action valence is a candidate mechanism for the influence of observer's internal states on properties experienced as phenomenally objective and yet loaded with meaning. 
1

2

3

4

5

6

7

8

9

10

11

12

13 Department of Life Sciences, Psychology Unit "Gaetano Kanizsa", 14 University of Trieste, Trieste, Italy.

${ }^{*}$ Corresponding Author: Carlo Fantoni

24 Department of Life Sciences

25

26

27 Via Weiss 21, 34128 Trieste (Italy)

28 email: cfantoni@units.it

29 phone: 00390405588871

30 fax: 00390405584220 

have a strong hedonic impact and affect how actors experience facial expressions of emotion. Using a novel Motor Action Mood Induction Procedure (MAMIP), they found consistent congruency effects in participants who performed a facial emotion identification task after a sequence of visually-guided reaches: a face perceived as neutral in a baseline condition appeared slightly happy after comfortable actions and slightly angry after uncomfortable actions. However, skeptics about the penetrability of perception (Zeimbekis \& Raftopoulos, 2015) would consider such evidence insufficient to demonstrate that observer's internal states induced by action comfort/discomfort affect perception in a top-down fashion. The action-modulated mood might have produced a back-end memory effect capable of affecting post-perceptual and decision processing, but not front-end perception.

Here, we present evidence that performing a facial emotion detection (not identification) task after MAMIP exhibits systematic mood-congruent sensitivity changes, rather than response bias changes attributable to cognitive set shifts; i.e., we show that observer's internal states induced by bodily action can modulate affective perception. The detection threshold for happiness was lower after fifty comfortable than uncomfortable reaches; while the detection threshold for anger was lower after fifty uncomfortable than comfortable reaches. Action valence induced an overall sensitivity improvement in detecting subtle variations of congruent facial expressions (happiness after positive comfortable actions, anger after negative uncomfortable actions), in the absence of significant response bias shifts. Notably, both comfortable and uncomfortable reaches impact sensitivity in an approximately symmetric way relative to a baseline inaction condition. All of these constitute compelling evidence of a genuine top-down effect on perception: specifically, facial expressions of emotion are penetrable by action-induced mood. Affective priming by action valence is a candidate mechanism for the influence of observer's internal states on properties experienced as phenomenally objective and yet loaded with meaning. 


\section{Introduction}

65 Penetrability of perception (Firestone \& Scholl, 2015; Zeimbekis \& Raftopoulos, 2015) refers 66 - among others - to the possible effects of bodily actions on affective perception (i.e., on aspects of perceived objects related to valence and arousal). Compared to the link between bodily actions (or intentions to activate the body; Vishton et al., 2007; Briscoe, 2014) and perception of spatial/material properties (Witt, 2011; Zadra \& Clore 2011), the link between bodily actions and affective perception appears quite plausible, being grounded in the phenomenal experience of bodily comfort/discomfort associated to motor actions. The emotional coloring of perception by observer's bodily actions could fall in the generic category of halo effects or attribution errors (consisting in, for instance, inappropriately attributing to external objects what should more reasonably pertain to the observing self).

Reading emotion in others' faces is a relevant instance of affective perception. However, despite the pervasive role of action in object representation (Santos \& Hood, 2009), little is known about the link between action and perception of facial expressions. This link is crucial in ontogenesis, as caregivers' responses to infant actions are the building blocks of socio-emotional development, visuo-motor control, and structuring of external object properties (Farroni, Csibra, Simion \& Johnson, 2002; Leppänen \& Nelson, 2009; Craighero, Leo, Umiltà \& Simion, 2011; Grossmann, Cross, Ticini \& Daum, 2013). As a case in point, the categorical perception and representation of emotionally expressive faces have been found to depend on bodily affective experience: children exposed to negative affective experience (e.g., physical abuse) over-identified anger relative to control children and produced discrimination peaks reflecting broader perceptual categorization of anger, relative to fear and sadness (Pollak \& Kistler, 2002). This is consistent with James-Lange theory of emotion (James, 1884) and recent evidence supporting the view that emotions are represented as culturally universal somatotopic maps; i.e., topographically distinct bodily sensations underlying the categorical perception of different emotions (Nummenmaa, Glerean, Hari \& Hietanen, 2014).

Bodily actions are both informative (about object properties) and affective, given that the experience of comfort/discomfort is a pervasive component of body feelings associated to action execution (Mark, et al., 1997), which can propagate from directly involved effectors to the whole 
93 body and transient mood (Cahour, 2008). In the workplace a posture that imposes musculoskeletal 94 discomfort has been found to induce a negative mood capable of impairing psychological health 95 (Conway, 1999). Body posture affects behavior through transient mood in other ways: for instance, 96 by modifying observers' judgment of neutral objects like ideographs (Cacioppo, Priester \& Berntson, 97 1993), as well as the subjective attitude towards dishonesty (Yap, Wazlawek, Lucas, Cuddy \& 98 Carney, 2013). Actions also manifest their effects on behavior implicitly, as revealed by the different 99 parameterization of hand movement kinematics in presence/absence of a social context (Becchio, 100 Sartori, Bulgheroni \& Castiello, 2008a; Becchio, Sartori, Bulgheroni \& Castiello, 2008b; Sartori, 101 Becchio, Bara \& Castiello 2009; Ferri, Campione, Dalla Volta, Gianelli \& Gentilucci, 2011; 102 Quesque, Lewkowicz, Delevoye-Turrell \& Coello, 2013; Fantoni, Rigutti, Piccoli \& Carnaghi, under 103 review; Straulino, Scaravilli \& Castiello, 2015), end-goal accuracy (Ansuini, Santello, Massaccesi, 104 Castiello, 2006), and motor affordances (Masson, Bub \& Breuer, 2011; Tucker \& Ellis, 1998; 105 Tipper, Howard \& Jackson, 1997; Glover, Rosenbaum, Graham \& Dixon, 2004) determined on the 106 basis of biomechanical compatibility, relative to size, shape, and material properties of the objecthand system (Mon-Williams \& Bingham, 2011; Flatters et al., 2012; Holt et al., 2013).

As regards the environment on which actions are directed to, while affective micro-valences are found in various types of everyday objects (Lebrecht, Bar, Barrett \& Tarr, 2012), faces of conspecifics are among the stimuli with the most extreme affective valences. Looking at emotional faces has indeed been shown to potentiate the sensory discrimination benefits of attention by 112 improving dimensions of early vision such as contrast sensitivity (Phelps, Ling \& Carrasco, 2006).

113 This is consistent with recent results suggesting that some components of facial expressions of 114 different emotions (e.g., widened/narrowed eyes in fear/disgust) involve different exposures of iris 115 and sclera that influence peripheral target discrimination by the expresser as well as the amount of 116 optical information available to the observer (Lee, Susskind \& Anderson, 2013).

117 Recently, Fantoni and Gerbino (2014; see also Fantoni, Cavallero \& Gerbino, 2014; Gerbino,

118 Fantoni, Nicolini, Volcic \& Domini, 2014; Gerbino, Manzini, Rigutti \& Fantoni, 2014) demonstrated 119 that the internal state of comfort/discomfort induced by reaching affects the identification of facial 120 expressions in a direction congruent with transient mood. To manipulate transient mood in a 121 controlled exploratory-action setting, Fantoni and Gerbino (2014; Gerbino et al., 2014) implemented 122 the Motor Action Mood Induction Procedure (MAMIP). The procedure required participants to 123 perform a series of either comfortable or uncomfortable visually-guided reaches of targets randomly 
124 located at short vs. long distances, corresponding to [0.65-0.75] vs. [0.9-1.00] ranges, relative to arm

125 length. Such a manipulation of distance was based on previous studies directly relating the subjective

126 state of comfort/discomfort to the individual reaching mode, with perceived discomfort increasing as

127 the number of body parts (muscles, joints) engaged in reaching increases (Mark et al., 1997).

128 The effectiveness of MAMIP was tested using a facial emotion identification task. After 129 having performed a sequence of mood-inducing actions (either comfortable or uncomfortable), 130 participants classified morphed faces displaying mixed expressions along the happy-to-angry 131 continuum as either "happy" or "angry". Systematic shifts of the point of subjective neutrality 132 between these facial expressions were fully consistent with a facilitation-by-congruency hypothesis: 133 comfortable actions increased the probability of classifying a neutral face as happy (positive 134 emotion); whereas uncomfortable actions increased the probability of classifying a neutral face as 135 angry (negative emotion). Furthermore, Fantoni and Gerbino (2014) found that JNDs were smaller 136 when facial emotion identification was preceded by reaching, relative to a baseline inaction 137 condition. They argued that hyperarousal from action (relative to inaction) improved the sensitivity 138 to subtle variations of facial expression and reduced the degree of classification uncertainty 139 (response times were shorter after reaching than in the baseline inaction condition). Finally, facial 140 emotion identification was more precise (lower JND) and faster after uncomfortable than 141 comfortable reaches, consistently with higher activation/arousal after uncomfortable motor actions.

142 This effects of motor action valence (in particular, comfort/discomfort of visually-guided

143 reaches) on perceived facial expressions fit well in the framework of the emotional mirror system 144 (Bastiaansen, Thioux \& Keysers, 2009) and are consistent with emotional response categorization 145 theory (Niedenthal, Halberstadt \& Innes-Ker, 1999), which implies that humans are tuned to respond 146 to conspecifics in a way that is congruent with their emotional state. Specifically, Fantoni and 147 Gerbino (2014) suggested that action comfort/discomfort may indirectly prime the successive 148 identification of emotions through transient mood induction (for a review of direct and indirect 149 affective priming effects see Janiszewski \& Wyer, 2014).

150 Expressive faces have indeed been used to demonstrate the occurrence of mood-dependent face 151 categorization using music as an inducer of internal states (Bouhuys, Bloem \& Groothuis, 1995).

152 Furthermore, the subjective state of comfort/discomfort has been found to correlate with mood 153 (Conway, 1999) and to depend upon the individual reaching mode, with discomfort being a direct 154 function of the amount of body movement supplementary to arm extension (Mark et al., 1997). The 
155 relationship between reaching distance and induced state of comfort/discomfort is mirrored by the 156 way the planning of hand movements with different movement amplitudes (involving variable 157 amounts of motor effort, muscular strength, and number of involved joints in the act) has been 158 recently shown to affect perception (Kirsch \& Kunde, 2013; Volcic, Fantoni, Caudek, Assad \& 159 Domini, 2013). Specifically, Kirsch and Kunde (2013) have shown that larger planning of goal160 directed hand movements obtained by instructing observers to perform movements with larger extent 161 causes the same spatial location to appear as further away. Similarly, Volcic et al. (2013) found that 162 the repeated execution of reaching movements with the visual feedback of the reaching finger 163 displaced farther in depth as if the actor would have a longer arm, enhanced both tactile sensitivity 164 and the perceived depth of disparity-defined objects.

165 However, no clear evidence allows us to decide whether changes in identification performance obtained by Fantoni and Gerbino (2014) depend on stimulus encoding, as involving a modification of the perceptual processing of facial emotion features, or response selection, as involving only a postperceptual modification of the response criterion and decision thresholds (Spruyt, Hermans, Houwer \& Eelen, 2002).

Are MAMIP effects truly perceptual?

The effectiveness of MAMIP has been tested by Fantoni and Gerbino (2014) under the assumption that the repeated execution of motor actions with a variable perceived degree of comfort generates a transient mood shift consistent with affective adaptation (Wilson \& Gilbert, 2008); i.e., with the progressive reduction of action valence paralleling a prolonged induction. Affective adaptation is a convenient umbrella expression, including anecdotal evidence that the valence of objects and events tends to fade away (i.e., tends to become less extreme) during prolonged exposure, as a possible consequence of a shift of the internal reference level involved in the evaluation of perceived objects and events. In the domain of motor actions, the repetition of comfortable reaches should: (i) induce a better mood in the actor; and (ii) make comfortable reaches progressively closer to neutrality (i.e., less discrepant from the shifted internal reference level). Likewise, the repetition of uncomfortable reaches should: (i) induce a worse mood in the actor; and

182 (ii) make uncomfortable reaches progressively closer to neutrality (i.e., less discrepant from the 183 shifted internal reference level).

184 Transient mood shifts modify the valence of not only bodily actions but also external objects; notably, expressive faces. Mood-congruent perception has been frequently reported in the literature 
186 (Bouhuys et al., 1995). Such an effect is consistent with the notion of affective priming (Klauer \& 187 Musch, 2003), given that the observer's state induced by action valence can be treated as an affective 188 prime, capable of pre-activating detectors selectively tuned to face features involved in the 189 expression of congruent emotions. Consider a morphed face that interpolates the facial expressions 190 of happiness and anger. Depending on observer's mood, the interpolated face will contain features 191 that support (in different proportions, by definition) both mood-congruent and mood-incongruent 192 emotions: for instance, a cheek puffer should be more salient than an upper lip riser for an observer in a positive mood, while the opposite should hold for an observer in a negative mood.

According to a strong version of the facilitation-by-congruency hypothesis, the selective preactivation of feature detectors by the transiently induced mood might produce two effects: an emotion identification bias (leading to a higher probability of classifying a neutral face as expressing a congruent emotion) as well as an improvement of the detection of a congruent emotion. According to a weak version of the same hypothesis, the transiently induced mood might produce only an emotion identification bias, without a change in sensitivity. This distinction is consistent with the categorization of priming effects proposed by Spruyt et al. (2002). According to the encoding stimulus account of affective priming, action valence acts as an affectively polarized prime that preactivates the memory representations of affectively related facial features, thus making it easier to encode emotional features belonging to the same valence domain rather than to a different one. Alternatively, according to the response selection account of affective priming action affects only post-perceptual processing by automatically triggering response tendencies that facilitate or interfere with response types. Notice that in both cases one should expect a shift of the point of subjective neutrality due to an unbalance that depends on the valence congruency between the action-induced mood and the facial expression of emotion. Both accounts predict that the likelihood of interpreting a facial expression as angry, for instance, is larger after an uncomfortable rather than comfortable reaching act.

211 Fantoni and Gerbino (2014) demonstrated the effectiveness of MAMIP using a facial emotion

212 identification (not detection) task. However, according to the above-described distinction and 213 following the criticism of identification data voiced by Firestone and Scholl (2015), results obtained

214 by Fantoni and Gerbino (2014) might simply reflect back-end memory processes rather than the 215 penetrability of perception by internal states. 
216 Furthermore, according to Fantoni and Gerbino (2014) the affective perception of faces is

217 influenced by congruency between valence of performed actions and valence of the target facial

218 expression (mediated by transient mood) as well as arousal. In principle, perceived facial expressions

219 might be influenced by both mood valence (negative after uncomfortable acts vs. positive after 220 comfortable acts) and arousal (higher after uncomfortable than comfortable acts). Independent of 221 congruency between observer's transient mood and emotion displayed by the target face, higher 222 arousal might have facilitated processing of expressive faces evaluated after uncomfortable actions.

223 The arousal-based hypothesis is consistent with evidence that visual sensitivity can be improved by 224 hyperarousal induced by cold pressor stimulation (Woods et al., 2012; Woods, Philbeck, \& Wirtz, 225 2013). In the experiment by Fantoni and Gerbino (2014) hyperarousal induced by uncomfortable 226 actions might have influenced emotion identification by modulating selective attention (Derryberry 227 \& Reed, 1998; Gasper \& Clore, 2002; Jeffries, Smilek, Eich, \& Enns, 2008). Following Firestone 228 and Scholl (2015), this interpretation would shed doubt on the truly perceptual origin of the effect 229 found by Fantoni and Gerbino (2014), as modulations of selective attention could in principle change 230 the input rather than subsequent processing.

231 To corroborate Fantoni and Gerbino's (2014) results, in the present study we asked whether 232 MAMIP effects reported in their paper are truly perceptual and whether evidence can be provided in 233 favor of an encoding rather than response account of affective priming of perceived facial 234 expressions of emotion, as mediated by action-induced mood states.

\section{Method}

\section{Rationale and expectations}

237 Experiments were designed to fulfil three goals: $(G 1)$ to disentangle the contributions of action 238 valence and arousal to the perception of facial expressions of emotion; (G2) to decide whether 239 action-induced mood congruency affects the valence of perceived expressions through a top-down 240 modulation of perceptual processing, signalled by a modification of emotion sensitivity in the 241 absence of response criterion shifts, or only affects a post-perceptual stage, by modifying the 242 response criterion (i.e., a decision, not perception, threshold); (G3) to evaluate the contribution of 243 action-induced mood congruency relative to the natural mood owned by a participant not performing 244 any movement before the perceptual task. 
To accomplish these goals we selected two emotions with opposite polarity in the valence

246

247

248

249

250

251

252

253

254

255

256

257

258

259

260

261

262

263

264

265

266

267

268

269

270

271

272

273 domain (happiness and anger), an objective yes/no task, and a sensitivity experiment (according to the terminology suggested by Witt, Taylor, Sugovic \& Wixted, 2015) comprising positive (signal + noise, $\mathrm{S}+\mathrm{N}$ ) trials in which the face displayed a target emotion and negative (noise, $\mathrm{N}$ ) trials in which the face displayed a neutral expression. Observers should be both fast and accurate when making their yes/no judgment on whether the face contained the emotion signal. Such a method minimized the role of cognitive top-down factors, unavoidable in the facial emotion identification procedure used by Fantoni and Gerbino (2014), and allowed us to decide whether MAMIP influenced emotion detection.

To measure sensitivity and bias in a facial emotion detection task we conducted four experiments with stimuli belonging to happiness and anger continua, generated by morphing a neutral face and an emotional face displaying either full happiness or full anger (Figure 1). Four morphing levels were chosen for each continuum, corresponding to different per cent emotion: 0 (original neutral face), 10, 20, and 30\% (Fig. 1B). Participants were tested individually in two successive sessions distinguished by reaches of opposite valence (comfortable/positive vs. uncomfortable/negative), with the ordering of action valences counterbalanced across participants. In doing so we disentangled the independent contribution of mood congruency, action valence, and arousal, by using as between-subjects factors the temporal ordering of action type (comfortable $\Rightarrow$ uncomfortable in Experiments 1 and 3 vs. uncomfortable $\Rightarrow$ comfortable in Experiments 2 and 4) and the valence of the target emotion (positive in Experiments $1 \& 2$ vs. negative in Experiments $3 \& 4$ ). By combining action valence and target emotion valence in a crossover design we planned to test the effect of action/emotion valence congruency on the detection of both positive and negative emotions (Fig. 1A, cells of the two matrices). Specifically, we planned to contrast:

- in Experiments $1 \&$ 2, happiness detection after congruent comfortable reaches (top-right cell of the left matrix \& top-left cell of the right matrix) vs. incongruent uncomfortable reaches (top-left cell of the left matrix \& top-right cell of the right matrix);

- in Experiments 3 \& 4, anger detection after congruent uncomfortable reaches (bottom-left cell of the left matrix \& bottom-right cell of the right matrix) vs. incongruent comfortable reaches (bottomright cell of the left matrix \& bottom-left cell of the right matrix). 
(A)

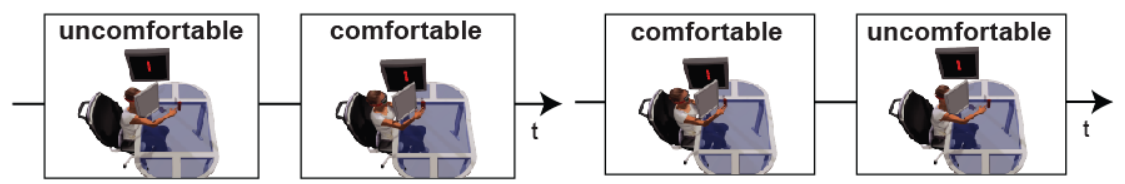

arousal by motor act

arousal by motor act

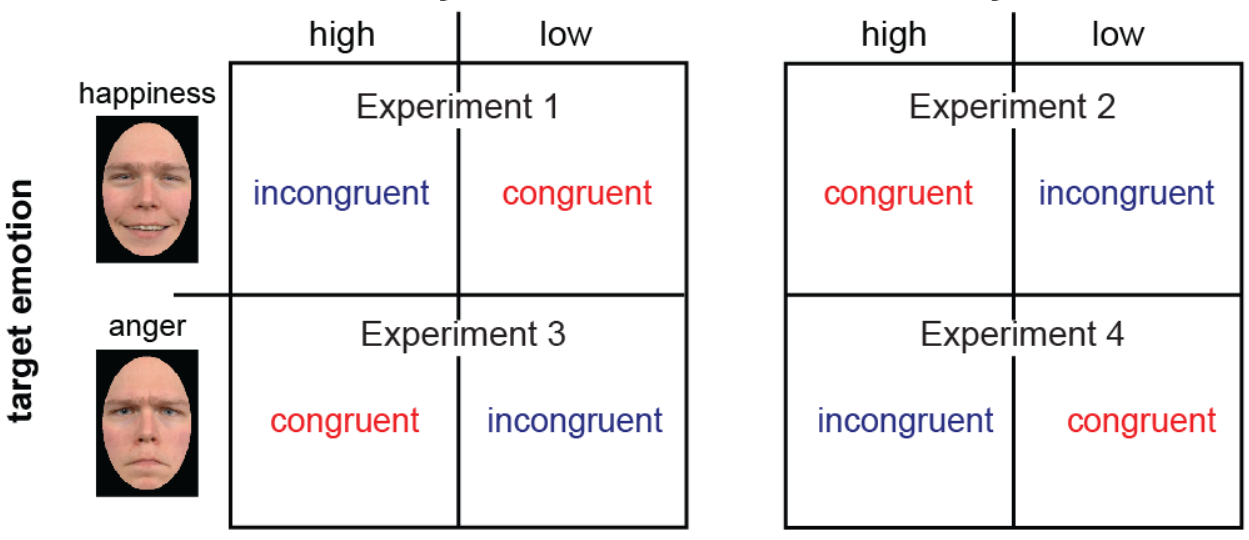

(B)

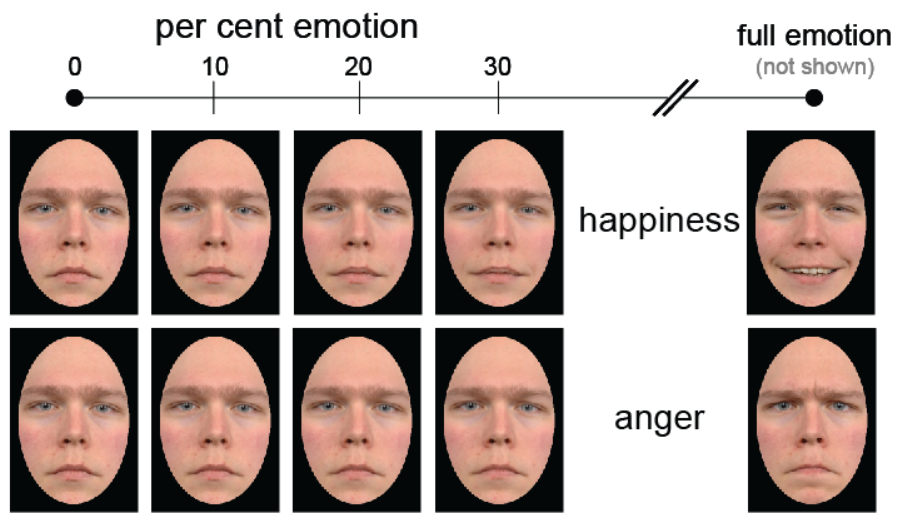

Figure 1. Experimental design and face set. Panel (A) illustrates the mixed factorial design used in our study. The two temporal sequences in the top row (uncomfortable $\Rightarrow$ comfortable on the left, comfortable $\Rightarrow$ uncomfortable on the right) represent the levels of the between-subjects factor Action Ordering. Matrix rows represent the levels (happiness/positive on top, anger/negative on bottom) of between-subjects factor Target Emotion. Each cell of the two matrices encodes the two levels of the within-subjects factor Congruency, based on the correspondence between the valence of bodily motor acts performed before the emotion detection task (same along columns) and the valence of target emotion (same along rows). Panel (B) shows the faces of neutral-happy (top) and neutral-angry (bottom) morph continua for an exemplar character (not used in our experiments) who gave permission for the usage of his image [see Fantoni \& Gerbino (2014, Fig. 1) for the characters of the Radboud database used in our experiments]. The panel illustrates, for each continuum, the four morph intensities $(0,10,20,30 \%)$ used in the present study and the full emotion face (right), used to generate the blended items by morphing it with the neutral face (left).

We used an equal-variance signal detection model (Macmillan \& Creelman, 2004) to obtain, proportions of yes responses in emotion present trials (hits) and emotion absent trials (false alarms). 
291 emotion) and absolute threshold $A T$ (defined as the per cent emotion value above which $d^{\prime}$ gets

292 positive). The third index, referring to the response stage, is criterion $c$.

293 After adaptation to comfortable/uncomfortable reaches under unrestrained body conditions, we

294 measured detection performance at three increasing levels of emotion $(10,20,30 \%)$ for two morph

295 continua (positive vs. negative target emotion), to contrast two hypotheses about possible effects of

296 motor action valence on sensitivity to facial expressions.

297 H1) According to the facilitation-by-congruency hypothesis (see Fig. 2), higher $d^{\prime}$ and lower $298 A T$ values were expected in congruent than incongruent conditions, independent of action valence 299 per se, as follows: (1) performing comfortable rather than uncomfortable actions should improve 300 happiness detection (Experiments 1 \& 2); (2) vice versa, performing uncomfortable rather than 301 comfortable actions should improve anger detection (Experiments $3 \& 4$ ). This hypothesis attributes 302 the role of mediator to a transient modification of mood in a direction congruent with the performed 303 action.

H2) According to the facilitation-by-arousal hypothesis, if uncomfortable reaches induce

305

306

307

308

309

310

311

312

313

314

315

316

317

318

319

320 higher arousal in the observer and emotion detection depends on arousal (at least more than on transient mood), higher $d^{\prime}$ and lower $A T$ values were expected after uncomfortable actions, regardless of target emotion valence, as follows: performing uncomfortable rather than comfortable actions should improve detection of both happiness (Experiments $1 \& 2$ ) and anger (Experiments $3 \& 4$ ).

Note that the two hypotheses lead to the same expectations for anger detection (Experiments 3 \& 4), but opposite expectations for happiness detection (Experiments $1 \& 2$ ). In the present study Action Ordering has been treated as a balancing between-subjects variable, while Target Emotion has been manipulated as a between-subjects factor to avoid a possible carryover effect intrinsic to an experimental design in which the same actors/observers detect emotions of opposite valence in successive sessions.

Furthermore, as depicted in Fig. 2 (panels A,D for happiness detection; panels B,E for anger detection), we considered two alternative possibilities about the MAMIP effect on $A T$, which was based on three $d^{\prime}$ values:

(1) additive effect, producing different $A T$ values in congruent (red solid line in Fig. 2A and 2B) and incongruent (cyan solid line in Fig. 2A and 2B) conditions, as a consequence of constant $d$ ' increments/decrements at increasing per cent emotion in the morphed target; an additive effect on $d^{\prime}$ 
321 would be signalled by significant main effects of morph intensity and of action/emotion valence

322 congruency, in the absence of their interaction, in all four experiments;

323 (2) multiplicative effect, producing the same $A T$ value in congruent (red solid line in Fig. 2D 324 and 2E) and incongruent (cyan solid line in Fig. 2D and 2E) conditions, as a consequence of 325 proportionally increasing $d$ ' increments/decrements as a function of per cent emotion in the morphed 326 target; a multiplicative effect on $d^{\prime}$ would be signalled by a significant morph intensity $\times$ 327 action/emotion valence congruency interaction in all four experiments.

328 Note that both hypotheses hold if the assumption of $d^{\prime}$ additivity is satisfied beyond MAMIP:

329 The expected increase of sensitivity as a function of per cent emotion in the morph (as calculated by 330 taking the proportion of false alarms in $\mathrm{N}$ trials as a common comparison value for the proportions of 331 hits associated to each tested level of per cent emotion in $\mathrm{S}+\mathrm{N}$ trials) should be approximately 332 linear, with slope $\beta_{2}$ and with negative intercept $\beta_{1}$, thus allowing for the identification of an absolute 333 threshold value above which $d^{\prime}$ gets positive even in a baseline inaction condition (grey dotted line in 334 Fig. 2).

335 Finally, we considered two alternative possibilities about the value of response criterion $c$, 336 calculated by pooling together hits and false alarms for all three morph intensities, within each block 337 of emotion detection trials following a MAMIP session. We expected that: (1) if MAMIP effects are 338 perceptual (i.e., if bodily actions penetrate affective perception) then the $c$ value should be constant 339 across action/emotion valence congruency conditions associated with mood-congruent sensitivity 340 effects, if bodily actions penetrate affective perception; (2) if MAMIP effects are post-perceptual the $341 c$ value should vary consistently with observer's transient mood, even in the absence of an effect on 342 sensitivity, due to a response bias in the direction of the action/mood congruent emotion (e.g., when 343 required to detect anger observers might increase their yes rate after uncomfortable, relative to 344 comfortable, actions). 

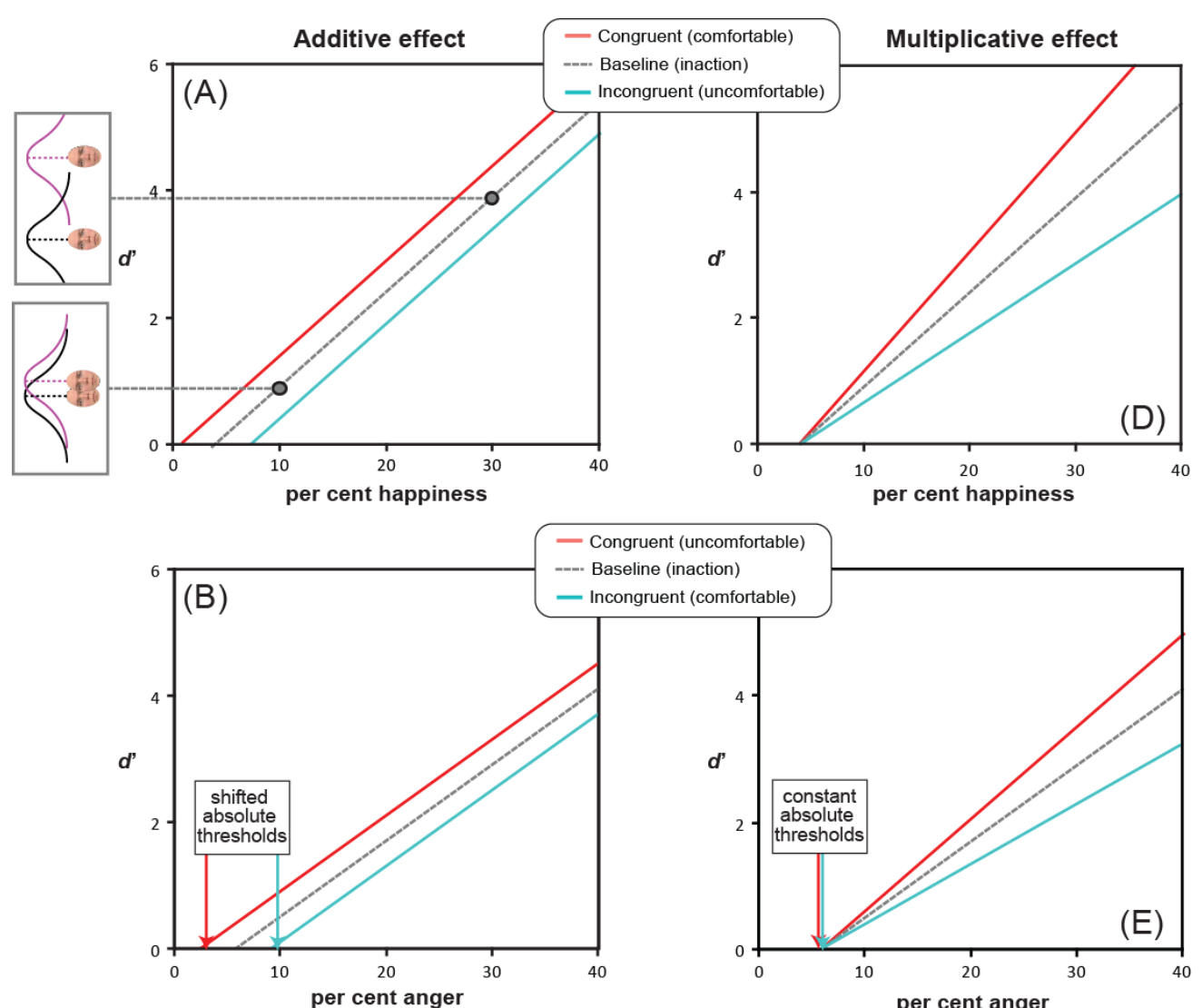

(uncomfortable)

Incongruent (comfortable)

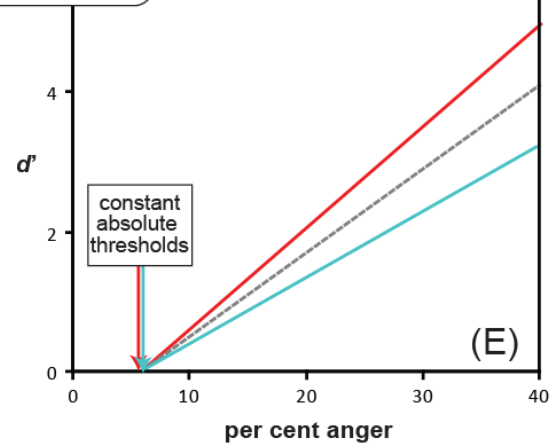

Emotion detection space

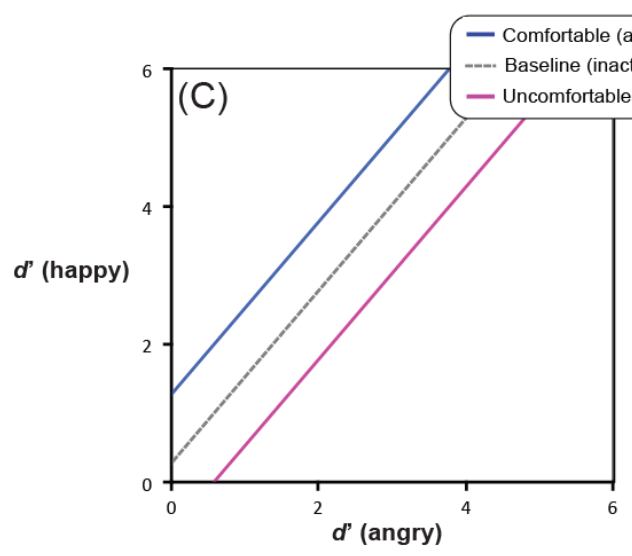

gry incongruent, happy congruent)

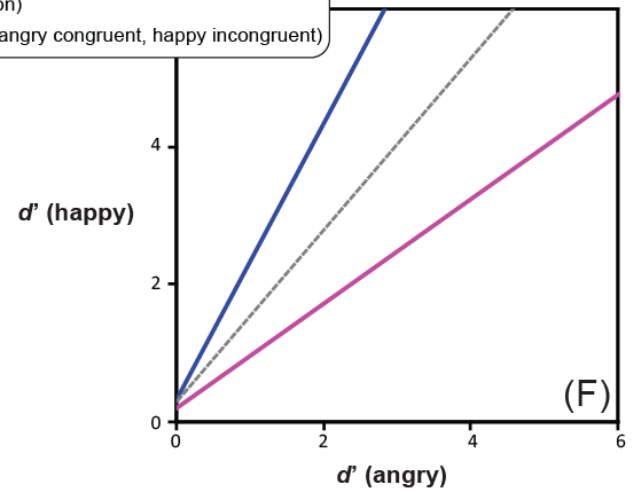

Figure 2. Alternative facilitation-by-congruency MAMIP effects. Predictions resulting from additive (panels A,B) vs. multiplicative (panels D,E) MAMIP effects are shown in the sensitivity-by-morph spaces where $d^{\prime}$ grows linearly as a function of per cent emotion in the morph for happy (panels A,D) and angry (panels B,E) faces. An additive MAMIP effect (A, B) is represented by parallel lines, with the red line (congruent/comfortable in A, and congruent/uncomfortable in $\mathrm{B}$ ) intersecting the per cent emotion axis at smaller values, than the cyan line (incongruent/uncomfortable in $\mathrm{A}$, and incongruent/comfortable in B): this produces a consistent shift in the absolute thresholds. A multiplicative MAMIP effect $(\mathrm{D}, \mathrm{E})$ is represented by lines diverging from a common origin lying along the per cent emotion axis that should produce constant absolute thresholds across different congruency conditions. The grey dotted line is the baseline performance if both comfortable and uncomfortable reaches impact sensitivity in a perfectly symmetric way. Predictions are recoded according to Equation 1 in the emotion detection space of panels C,D. The blue solid line stands for the line of comfortable reaches (happy congruent $d^{\prime}$ as a function of angry incongruent $d^{\prime}$ ); the violet solid line stands for the line of uncomfortable reaches (happy incongruent $d^{\prime}$ as a function of angry congruent $d^{\prime}$ ). 
358 Comparison with a baseline inaction condition

359 To accomplish G3 we compared the emotion sensitivity in Experiments 1-4 (after MAMIP)

360 with that obtained in a preliminary experiment (baseline inaction condition, see Preliminary

361 experiment section) on a large group of psychology undergraduates $(\mathrm{N}=91,66$ females) who

362 performed a $2 \mathrm{AFC}$ task not preceded by any motor task. Each $2 \mathrm{AFC}$ trial of the preliminary

363 experiment included a neutral face and a morphed face with $15 \%$ emotion (anger or happiness).

364 Participants should indicate which face (left/right) displayed an emotion.

We considered two possible outcomes of the comparison between Experiments 1-4 and the 366 preliminary experiment, depending on whether the effect of action-induced mood on yes/no emotion sensitivity was symmetric or asymmetric, relative to the baseline inaction condition in which the mood was expected to be neutral (Fig. 2C, 2F). Consider comfortable reaches. If comfort empowers our sense of motor skillfulness, thus contributing to the establishment of a more positive mood than the one experienced in the baseline inaction condition, then in Experiments $1 \& 2$ happiness sensitivity after comfortable reaches (congruent condition) should be higher than happiness sensitivity in the preliminary experiment; conversely, in Experiments $3 \& 4$ anger sensitivity after comfortable reaches (incongruent condition) should be lower than anger sensitivity in the preliminary experiment. This hypothesis is based on the general idea that actions executed within the comfort range are rewarding and engagement in comfortable actions is more pleasant than comfort associated to inaction. Consider now reaching outside the natural grasping range, which should induce a negative mood as a direct product of discomfort or as an effect of experiential avoidance (Sloan, 2004). In Experiments $3 \& 4$ anger sensitivity after uncomfortable reaches (congruent condition) should be higher than anger sensitivity in the preliminary experiment, while in Experiments $1 \& 2$ happiness sensitivity after uncomfortable reaches (incongruent condition) should be lower than happiness sensitivity in the preliminary experiment.

A perfectly symmetric facilitation-by-congruency effect of action-induced mood on emotion sensitivity is illustrated in Fig. 2 according to additive (Fig. 2A, 2B) and multiplicative (Fig. 2D, 2E) models. The grey dotted line provides a reference along which the baseline $d^{\prime}$ is expected to lie on. Considering a generic emotion detection Cartesian space with happiness $d^{\prime}$ on the $y$ axis and anger $d^{\prime}$ on the $x$ axis (Fig. 2C, 2F), this hypothesis leads to the following general expectation, irrespective on whether the MAMIP effect is additive (Fig. 2C) or multiplicative (Fig. 2F): the point representing performance in the baseline condition should lie in the portion of space between the blue solid line, 
389 depicting sensitivity after comfortable reaches (with anger sensitivity in the incongruent condition on

390 the $x$ axis and happiness sensitivity in the congruent condition on the $y$ axis), and the violet solid line,

391 depicting sensitivity after uncomfortable reaches (with anger sensitivity in the congruent condition

392 on the $x$ axis and happiness sensitivity in the incongruent condition on the $y$ axis). These two lines

393 can be recovered combining intercepts and slopes of the pair of lines best fitting the $d^{\prime}$ triplets over

394 the per cent emotion in the morph associated to comfortable (happy congruent and angry incongruent

395 lines) and uncomfortable (happy incongruent and angry congruent lines) reaches. It can indeed be

396 shown that slope $\beta_{2 \mathrm{G}}$ and intercept $\beta_{1 \mathrm{G}}$ of a line in the generic emotion detection space are given by:

397

$$
\beta_{1 G}=\beta_{1 \text { happy }}-\frac{\beta_{2_{\text {happy }}} \beta_{1 \text { angry }}}{\beta_{2 \text { angry }}}
$$

$$
\beta_{{ }_{G}}=\frac{\beta_{2 \text { happy }}}{\beta_{2 \text { angry }}}
$$

Equation 1

where $\beta_{1 \text { happy }}$ and $\beta_{2 \text { angry }}$ corresponds to the intercept and slope of the lines best fitting the average $d^{\prime}$ triplets over morph intensity for happiness and anger sensitivity after mood-congruent and mood-incongruent reaches for the comfortable blue solid line, and vice versa for the uncomfortable violet solid line of Fig. 2C and 2F.

If the facilitation-by-congruency effect of action-induced mood on emotion sensitivity is perfectly symmetric, as exemplified in Fig. 2, then the baseline $d^{\prime}$ should lie along the grey dotted line of either Fig. 2C, in the case of an additive effect, or Fig. 2F, in the case of a multiplicative effect, with the parameters of the grey dotted line resulting from averaging the parameters of the comfortable and uncomfortable lines. Otherwise, if the facilitation-by-congruency effect is asymmetric - possibly because comfortable reaches are equivalent to neutral inaction and do not modify the observer's mood - then the baseline $d^{\prime}$ should lie along the blue line of either Fig. 2C, in the case of an additive effect, or Fig. 2F, in the case of a multiplicative effect.

\section{Participants}

Forty undergraduates of the University of Trieste, all right handed, participated in the 412 experiment lasting about one hour and half. All had normal or corrected-to-normal vision, were 413 naïve to the purpose of the experiment, had no history of mood disorder and were not using 414 antidepressant medications at the moment of the experiment. They were randomly assigned to one of 415 the four experiments resulting from the combination of Action Ordering (comfortable first, 
416 uncomfortable first) and Target Emotion (happiness, anger). Each experiment included 10

417 participants distributed as follows across females and males: Experiment 1 (uncomfortable

$418 \Rightarrow$ comfortable, happiness; mean age $=23.7, \mathrm{SD}=2.5$ ), females $=7$; Experiments 2 (comfortable

$419 \Rightarrow$ uncomfortable, happiness; mean age $=24.3, \mathrm{SD}=2.1$ ), females $=6$, Experiment 3

420 (uncomfortable $\Rightarrow$ comfortable, anger; mean age $=23.2, \mathrm{SD}=2.1$ ), females $=6$; Experiment 4

421 (comfortable $\Rightarrow$ uncomfortable, anger; mean age $=23.5, \mathrm{SD}=1.5$ ), females $=7$.

422 The study was approved by the Research Ethics Committee of the University of Trieste 423 (approval number 52) in compliance with national legislation, the Ethical Code of the Italian 424 Association of Psychology, and the Code of Ethical Principles for Medical Research Involving 425 Human Subjects of the World Medical Association (Declaration of Helsinki). Participants provided 426 their written informed consent prior to inclusion in the study. The Ethics Committee of the 427 University of Trieste approved the participation of regularly enrolled students to data collection 428 sessions connected to this specific study, as well as the informed consent form that participants were 429 required to sign. Dataset is available as a Supplemental data file (Supplemental_Dataset_S1)

\section{Apparatus, stimuli \& design}

431 The experimental setting utilized exactly the same Augmented Reality apparatus described in 432 Fantoni and Gerbino (2014). Participants were seated in a dark laboratory in front of a high-quality, 433 front-silvered $40 \times 30 \mathrm{~cm}$ mirror, slanted at $45^{\circ}$ relative to the participant's sagittal body midline and 434 reflecting images displayed on a Sony Trinitron Color Graphic Display GDM-F520 CRT monitor 435 (19"; $1024 \times 768$ pixels; $85 \mathrm{~Hz}$ refresh rate), placed at the left of the mirror (Fantoni \& Gerbino, 436 2014, Fig. 1b, c).

437 The same 3D visual displays used by Fantoni and Gerbino (2014) were used in the MAMIP 438 reaching phases of the four experiments: (1) a high-contrast vertically oriented random-dot rod (30\% 439 density, visible back-surface, $7.5 \mathrm{~mm}$ radius, $65 \mathrm{~mm}$ height), depicted in Fig. 3A (see for details 440 Fantoni \& Gerbino, 2014, Fig. 1a); (2) a virtual red sphere (3 $\mathrm{mm}$ diameter) that visually marked the 441 tip of the participant's index finger in 3D space after the finger departed from its starting position of 442 about $30 \mathrm{~mm}$. Both stimuli were rendered in stereo and were generated using a frame interlacing 443 technique in conjunction with liquid crystal FE-1 goggles (Cambridge Research Systems, 444 Cambridge, UK) synchronized with the monitor's frame rate, and were updated in real time with 445 head and hand movements (acquired on-line with sub-millimeter resolution by using an Optotrak 
446 Certus with one position sensor) so to keep their geometrical projection always consistent with the 447 participant's viewpoint.

448 Each reaching session was preceded by the same calibration procedure used in Fantoni and 449 Gerbino (2014), and detailed in Nicolini, Fantoni, Mancuso, Volcic and Domini (2014). In particular 450 the position of the index tip and of the eyes (viewpoint) were calculated during the system 451 calibration phase using three infrared-emitting diodes firmly attached on the distal phalanx and on 452 the back of the head. This was needed to ensure a correct geometrical projection of the 3D visual 453 displays (virtual rod and index tip) according to the shifts of different body segments. Specifically, 454 head movements updated the participant's viewpoint to present the correct geometrical projection of 455 the stimulus in real time. A custom made Visual $\mathrm{C}++$ program supported stimulus presentation and 456 the acquisition of kinematic data associated to the reaching phase, as well as the recording of yes/no 457 responses relative to the facial emotion detection task (left/right keys of the computer keyboard) and 458 RTs.

The simulated egocentric depth of the rod along the line of sight was manipulated according to 460 empirical data by Fantoni \& Gerbino (2014, but see also Mark et al., 1997) Fantoni \& Gerbino, 461 (2014) asked observers to rate the discomfort of 50 reaches whose depth was randomly varied across 462 trials in the entire [0.65-1.00] range of arm length using a 0-50 discomfort scale adapted from the 463 pain scale by Ellermeier, Westphal \& Heidenfelder (1991). Following their results the simulated 464 egocentric depth of the rod axis along the line of sight was randomly chosen within the [0.65-0.75] 465 range for the comfortable-reaching block and within the [0.90-1.00] range for the uncomfortable466 reaching block, relative to the arm length of each participant.

467 Furthermore, a physical rod (equal in shape to the virtual one) placed behind the mirror that 468 fully occluded it was attached to a linear positioning stage (Velmex Inc., Bloomfield, NY, USA). 469 The position of the physical rod was matched to the egocentric depth of the simulated rod on a trial470 by-trial basis, with submillimiter precision (straight-line accuracy $=0.076 \mathrm{~mm}$ ) with the Velmex 471 motorized Bslides assembly (Bloomfield, NY, USA), so that real and virtual stimuli were perfectly 472 aligned. This provided participants with a fully consistent haptic feedback as the red sphere marking 473 the index tip reached the illusory surface defined by the constellation of random dots shaping the 474 virtual rod exactly when the participant's finger entered in contact with the real rod. Furthermore to 475 ensure consistent vergence and accommodative information, the position of the monitor was also 476 attached to a Velmex linear positioning stage that was adjusted on a trial-by-trial basis to equal the 
477 distance from the participant's eyes to the virtual/real object that should be reached during the 478 reaching block. Synchronizing stimulus presentation with the motorized linear positioning stage 479 system allowed us to randomly manipulate the distance of the reaches in a rather continuous way 480 (step resolution $=0.076 \mathrm{~mm}$ ) over the depth ranges used in our study; i.e., in both comfortable 481 ([0.65-0.75] the arm length) and uncomfortable ([0.75-1.00] the arm length) reaching blocks.

482 The facial stimulus set included the same 8 characters (four Caucasian males and four 483 Caucasian females) that Fantoni and Gerbino (2014) selected from the Radboud University 484 Nijmegen set (Langner et al., 2010): namely, female models 1, 4, 14, 19; and male models 20, 30, 485 46, 71. For each of the 8 selected characters of the Radboud database (included in Fantoni \& 486 Gerbino, 2014, Fig. 1) we utilized color photographs displaying faces expressing two basic emotions, 487 happiness and anger, and the corresponding neutral faces (all stimuli produced a high agreement with 488 intended expressions in the validation study). A neutral-to-angry and a neutral-to-happy continua were first generated for each of the 8 characters, morphing the fully angry/happy face and the neutral face in variable proportions, in 5 per cent steps, using MATLAB software adapted from open source programs. For every two pairs of facial images we selected about 75 key points. The software generated a synthetic image containing a specified mixture of the original expressions, using a sophisticated morphing algorithm that implements the principles described by (Benson \& Perrett 1999). As in Marneweck, Loftus \& Hammond (2013) and Fantoni and Gerbino (2014), we identified corresponding points in the two faces, with more points around areas of greater change with increasing emotional intensity (pupils, eyelids, eyebrows, and lips). Then, as depicted in Fig. 1B, for every character we selected three target stimuli for the neutral-to-angry continuum and three target stimuli for the neutral-to-happy continuum, corresponding to the following morph intensities: $10 \%$ emotion ( $90 \%$ neutral), $20 \%$ emotion ( $80 \%$ neutral), 30\% emotion (70\% neutral). Neutral stimuli were the original "no emotion" faces of the 8 selected characters. All images were aligned for facial landmarks and masked by an oval vignette hiding hair and ears presented on a black surround, the

502 vignette being centered on the screen and occupying a visual size of $7.5^{\circ} \times 10.7^{\circ}$ at the viewing 503 distance of $50 \mathrm{~cm}$.

504 The target emotion was positive (happiness) in Experiments $1 \& 2$ vs. negative (anger) in

505 Experiments 3 \& 4. Each experiment included two random sequences (one for each MAMIP 506 condition, comfortable vs. uncomfortable) of 32 facial images resulting from the product of 8 
507 characters $\times 4$ morph intensities (including the neutral face corresponding to $0 \%$ emotion in the 508 morph).

509 The complete $2 \times 2 \times 2 \times 4$ mixed factorial design shown in Fig. 1A included the two between510 subjects factors called Action Ordering (comfortable $\Rightarrow$ uncomfortable vs. uncomfortable $511 \Rightarrow$ comfortable, Fig. 1A, top row) and Target Emotion (positive vs. negative, Fig. 1A, matrix rows) 512 and the two within-subjects factors called action/emotion valence Congruency (congruent vs. 513 incongruent, Fig. 1A, matrix columns, depending on encoding in each cell) and Morph Intensity (0, $51410,20,30 \%$ emotion in the morph, Fig. 1B).

\section{Preliminary experiment}

516 The values of morph intensities used for the target stimuli in our two emotion continua were

517 established empirically on the basis of the results of the preliminary baseline experiment. The 518 instructor (author WG) explained that in each trial two faces (one neutral and one displaying an 519 emotion) would be presented (1500 ms exposure) on the left/right of a continuously visible central 520 fixation cross. Then, he explained that the positions of the two faces were randomized and that the 521 task consisted in writing the letter S (sinistra, left) or D (destra, right) in the appropriate cell of the 522 response sheet, to indicate the position of the face expressing the emotion. The response sheet 523 required participants to answer preliminary questions about their gender and age, and to fill 32 cells 524 (16 for each of the two experimental blocks).

525 Then, the instructor presented a sample of 8 trials, to familiarize participants with all 526 characters: 4 practice trials included a morphed happy face and 4 a morphed angry face. The experimental session included two blocks of 16 trials each. The first block included a random sequence of trials in which a neutral face was shown together with a 15\% happy face ( 8 characters by two positions); the second block included a different random sequence of trials in which a neutral face was shown together with a 15\% angry face (always balancing characters and positions). To prevent mistakes in filling in the response sheet, the instructor named the trial number aloud, before the presentation of the $300 \mathrm{~ms}$ fixation dot preceding stimulus presentation.

Stimuli were presented using PowerPoint through a high resolution MARCA video projector 534 connected to the graphic output of MAC-PRO (3D graphic accelerator). Participants were comfortably seated in a dimly lit classroom while facing the projection screen at the average distance of $12.25 \mathrm{~m}$ away. The average visual angle subtended by classroom displays was similar to the visual 
537 angle in Experiments 1-4, given that they were 35 times larger than the stimuli displayed on the lab

538 CRT and the participant's distance from the projection screen was about 35 times the one in the lab.

539 We extracted individual $d$ ' values from raw proportions of "left" responses out of 8 trials, for

540 both hits and false alarms, using a $g l m$ with variable intercept $\beta_{1}$ and slope $\beta_{2}$ for every participant

541 and emotion condition (Knoblauch \& Maloney, 2012). This corresponded to reparametrize each

542 individual Gaussian function fit in term of $\beta_{2} / \sqrt{2}$, or the $d^{n}$ for a $2 \mathrm{AFC}$ paradigm (i.e., the difference

543 between hit and false alarm rates on the probit scale).

544 From the analysis of valid $d$ ' values (those between \pm 2.5 individual standard deviation from

545 the mean which led to the removal of $5 d^{\prime}$ values from the negative and $4 d^{\prime}$ values from the positive 546 target emotion condition, each out of the 91 values collected over the two entire emotion conditions)

547 two main results are: (1) the $15 \%$ morph level produced a sizable perceptual effect on emotion 548 detection eliciting a non null sensitivity for both positive $(1.26 \pm 0.159$, two-tailed $t$ vs. $0=7.90, d f=$ $54986, p<0.001, d=1.70)$ and negative $(0.15 \pm 0.049$, two-tailed $t$ vs. $0=2.96, d f=85, p=0.0039, d=$ 550 0.64) target emotions; (2) emotion detection as elicited by our stimulus set was anisotropic as 551 revealed by the lower average sensitivity in the anger rather than in the happiness detection task 552 (Welch two sample $t=6.64, d f=171, p<0.001, d=1.01$ ).

553 These preliminary results were in agreement with previous results in the emotion perception 554 literature showing that realistic faces, as those used in the current experiments, often give rise to a 555 happiness (rather than the more often found anger) detection advantage relative to both angry 556 (Becker, Anderson, Mortensen, Neufeld \& Neel, 2011; Becker, Neel, Srinivasan, Neufeld, Kumar \& 557 Fouse, 2012; Juth, Lundqvist, Karlsson \& Ohman, 2005) and sad (Srivastava \& Srinivasan, 2010) 558 faces. Furthermore, the results demonstrated that morphing from our face set in a range above and 559 slightly below the $15 \%$ should elicit sizable sensitivity differences with both positive and negative emotions, thus setting the optimal conditions for measuring the effect of bodily action on the perception of emotion. Finally, the average $d^{\prime}$ values obtained with such a large sample of observers is representative of emotion detection performance in a baseline inaction condition and can thus be used as a reference value to evaluate whether, according to G3 (see also Fig. 2), the effect of MAMIP is symmetric or not (results in Fig. 7).

\section{Procedure}

As shown in Fig. 3, our procedure included two sessions, each composed by an induction phase (MAMIP reaching phase) followed by a test phase (emotion detection task). 


\section{Calibration/positioning of} linear actuators

(A)

(B)

570

571

572

573

574

575

576

577

578

579

580

581

582

583

584

585

586

587

588

589 of the test phase (facial emotion detection task).

\section{Calibration/positioning of} linear actuators

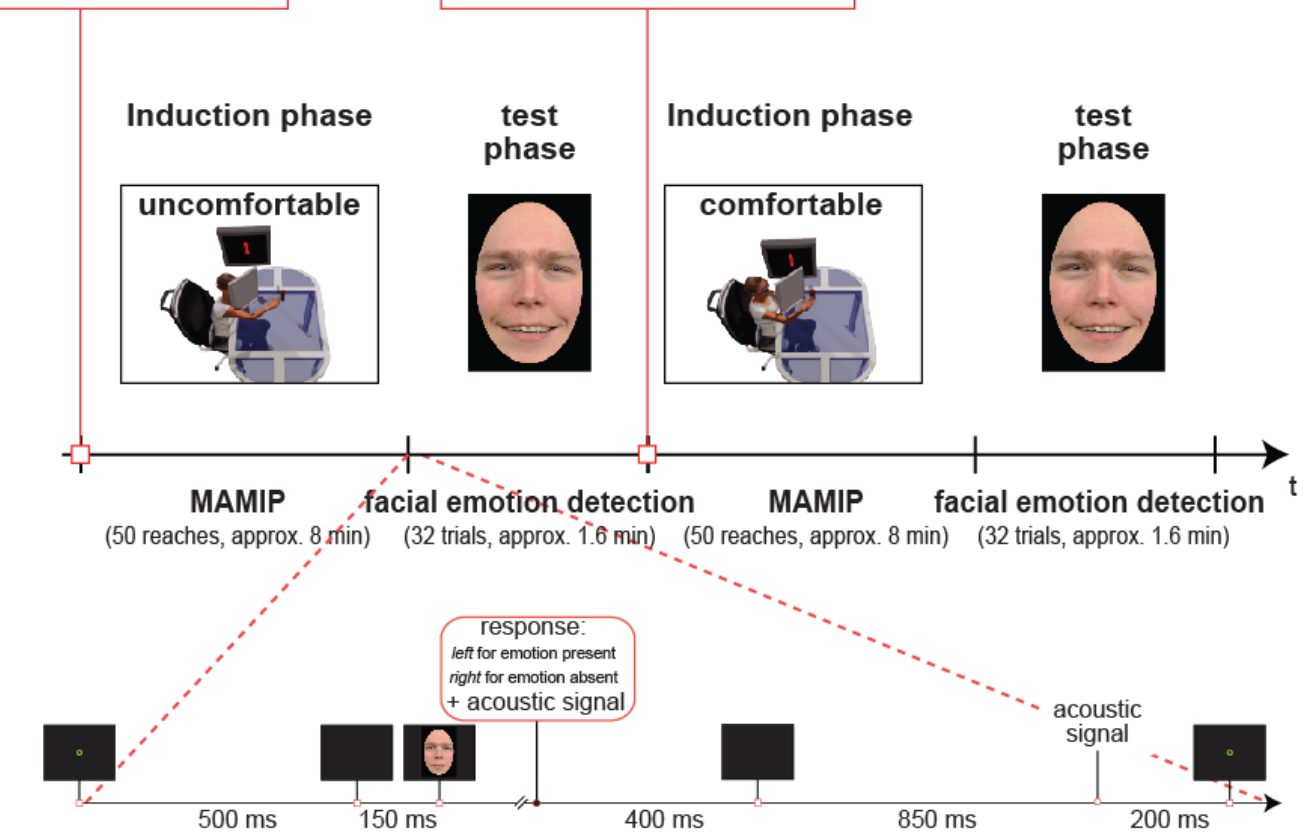

Figure 3. Temporal sequence of phases in our Experiments (A) and details of the facial emotion detection task (B). (A) Two blocks of Experiment 1 separated by the calibration phase. In the first induction phase the valence of the motor action is negative, and the test phase requires the detection of a face displaying an incongruent positive emotion. In the second induction phase the valence of the motor action is instead positive, and the test phase requires the detection of a face displaying a congruent positive emotion. The inset in (B) shows the detailed temporal sequence of events in one trial

MAMIP reaching phase. To maximize data comparability the present study followed the original procedure implemented by Fantoni and Gerbino (2014; see their procedure section for details). Participants were asked to perform 50 successive unrestrained reaches towards a virtual random-dot rod positioned along the line of sight at a variable depth randomly selected with submillimiter precision $(0.076 \mathrm{~mm})$ within the [0.65-0.75] range of individual arm length for the Comfortable session and within the [0.90-1.00] range of individual arm length for the Uncomfortable session. According to Mark et al. (1997) leaving unrestraining the body during reaches allows the number of degrees of freedom involved in the motor act to vary consistently with the depth of the reach, that in turn should be a prerequisite for the establishment of a concurrent variation of comfort/discomfort. Participants were instructed to perform the movement in a rather natural way (neither too slowly nor to fast), to rest with their index in contact with the real rod until the disappearance of the stimulus, and then to move back their hand in the starting position.

The finger movement started in full darkness from a fixed out-of-view position shifted relative to the body midline by about $25 \mathrm{~cm}$ from the sagittal plane and $15 \mathrm{~cm}$ from the coronal plane. This 
590 position was registered at the end of the system calibration phase after which the linear positioning 591 stages for the monitor and for the real rod moved for about $1000 \mathrm{~ms}$. At the end of such positioning 592 phase an acoustic feedback $(200 \mathrm{~Hz}$, lasting $200 \mathrm{~ms})$ signalled that the observer could start her 593 movement. The virtual rod and virtual red sphere marking the fingertip were visible for about $3.5 \mathrm{~s}$ 594 from the moment the finger entered in the participant's visual field after a shift of about $3 \mathrm{~cm}$ from 595 its starting position. The end of the reaching movement was accompanied by a haptic feedback and 596 was followed (after a variable lapse of time depending on individual reaching velocity) by the same 597 acoustic signal after which the simulated rod disappeared for about $1000 \mathrm{~ms}$. As soon as the 598 simulated rod disappeared the observer was asked to place his hand again in the starting position. At 599 the end of the blank period the motorized positioning stages were activated so to adjust the position 600 of the physical rod behind the mirror to the egocentric depth of the successive simulated depth rod. 601 At the end of the motor movements (after $3000 \mathrm{~ms}$ ) a second acoustic signal (200 ms) was provided 602 to the observer signalling that she could start the hand movement. Successful reaches were ensured 603 by providing the observer with a fully consistent haptic feedback at the end of each reaching act. 604 This was possible thanks to the motorized positioning system used to perfectly align on a trial by 605 trial basis the position of the physical rod to the one of the simulated rod. Furthermore, reaching 606 kinematics and head position were on-line monitored by the experimenter from a remote station 607 invisible to the observer.

608 Each reaching session lasted a total of $8 \mathrm{~min}$ on average, subdivided as follows: $4.3 \mathrm{~min}$ of 609 forward reaching actions +0.83 min of backward reaching actions +2.83 min death times (including 610 the time of motion of linear positioning stages and of acoustic signals). The forward reaching acts 611 can be described as composed by three successive phases: (1) a movement planning phase (from the 612 acoustic signal to the appearance of the virtual random dot, lasting $1.67 \pm 0.18 \mathrm{~s})$; (2) an execution 613 phase (from the appearance of the virtual random dot to the time of index contact with the real rod, 614 lasting $2.09 \pm 0.07 \mathrm{~s}$ for long uncomfortable and $1.59 \pm 0.06 \mathrm{~s}$ for short comfortable reaches); (3) an 615 exploration phase (from finger contact until the disappearance of the object, lasting $1.41 \pm 0.07 \mathrm{~s}$ for 616 long uncomfortable and $1.91 \pm 0.06 \mathrm{~s}$ for short comfortable reaches).

617 The procedure included: a session in which the participant's arm length at rest (i.e., the 618 effective maximum reach) was carefully measured following a procedure similar to the one used by 619 Mark et al. (1997, see Appendix 1A), instructions, a training with 15 reaches randomly extracted 620 across the entire depth range used in the experiment (0.65-1.00 of arm length), and the experimental 
621 session. We decided to eliminate participant that during the training session were unable to perform a 622 sequence of at least 5 successful reaches (getting in contact with the real rod within the predefined 623 temporal interval of $3.5 \mathrm{~s}$ ) in the last 8 trials. No participants were excluded on the basis of this 624 action criterion.

625 Facial emotion detection (yes/no task) phase. Participants completed a randomly ordered block 626 of 32 facial emotion detection trials just after each reaching session. Each block resulted from the 627 combination of 8 characters ( 4 actors and 4 actresses) $\times 4$ morph intensities $(0,10,20,30 \%$ emotion 628 in the morph), with an overall $3: 1$ ratio of $[\mathrm{S}+\mathrm{N}]$ to $[\mathrm{N}]$ trials. In other terms, every stimulus was 629 presented to participants only once, to minimize possible attempts to reproduce responses already 630 given to stimuli remembered as identical to the target and to keep the test phase reasonably short, 631 compared to duration of the induction phase. The target emotion was happiness in Experiments $1 \&$ 6322 and anger in Experiments $3 \& 4$.

633 The same yes/no task was applied in all four experiments. At the beginning of each emotion 634 detection trial a 30-pixel-wide green fixation circle was displayed at the center of the screen for 635 about $500 \mathrm{~ms}$ (Fig. 3B). This was substituted by a brief refreshing blank screen of about $150 \mathrm{~ms}$. The 636 face stimulus was then displayed until the participant pressed one of two response keys with his/her 637 left hand: left key for yes ("Emotion present") vs. right key for no ("Emotion absent"). The default 638 minimum duration of the face stimulus was $400 \mathrm{~ms}$. After key press, a low tone lasting $400 \mathrm{~ms}$ 639 signalled the response recording and a blank screen lasting about $850 \mathrm{~ms}$ followed. The end of such a 640 blank screen period was signalled by a mid tone acoustic feedback lasting $200 \mathrm{~ms}$. The next trial was 641 thus presented. Notice that the left hand was used for responses to the emotion detection task while 642 the right hand, wearing markers, was resting after the reaching session.

643 On average each facial emotion detection session lasted a total of 1.6 min subdivided as 644 follows: $0.6 \mathrm{~min}$ of response/observation time $(32$ trials $\times$ average OT $=1154 \pm 14 \mathrm{~ms})+1.0 \mathrm{~min}$ of 645 rest times (including the timing of fixation, acoustic signals and blank screen period).

646 The procedure included: (a) instructions; (b) a session of familiarization with the face set 647 (including a serial presentation of the 32 facial stimuli to be presented in the facial emotion detection 648 task, ordered by character and morph intensity); (c) a training block of 32 emotion detection trials (in 649 which the face of each of the 8 characters was presented four times, one in the neutral pose and three 650 times as a 50\% morph of the emotion appropriate for each experiment); (d) the experimental session. 651 The training block was designed having in mind two goals: (a) familiarization with neutral faces, [N] 
652 trials, and with the target emotion, when its intensity was well above the levels utilized in $[\mathrm{S}+\mathrm{N}]$ 653 trials; (b) elimination of participants with an inadequate level of sensitivity. Only participants with 654 more than $90 \%$ correct responses during training entered the experimental session (four participants 655 excluded). Written instructions required participants to use the green circle to support steady fixation 656 during stimulus presentation, to keep in mind that just one fourth of the stimuli displayed a neutral 657 facial expression, and to be fast and accurate, considering that stimulus presentation was terminated 658 by the response. We chose this option, rather than a fixed exposure time, to account for individual 659 variability in the processing of different emotions and to allow observers to modulate the amount of 660 time in which stimulus-driven emotional information was available. The response-terminated 661 presentation method sets the conditions for: (1) a trade-off between individual $d^{\prime}$ and 662 response/observation time (OT); (2) an inverse modulation of OT as a function of the intensity of the 663 signal in $[S+N]$ trials (Fantoni, Gerbino \& Kellman, 2008; Gratton, Coles, Sirevaag, Eriksen, \& 664 Donchin, 1988; Wickens, 1984).

We ran a preliminary lme analysis on the relationship between individual $d^{\prime}$ values (see the Statistical analysis section for details on their computation) and response speed, computed as the inverse of OT values for correct responses (i.e., 1000/OT in the interval between $\pm 2.5 \mathrm{SD}$ from the individual mean, which led to the removal of 27 out of 2496 total trials). Response speed values were averaged within all cells of the overall experimental design. The lme analysis revealed: (1) a weak speed-sensitivity trade-off, given that the main effect of response speed on $d^{\prime}$ was marginal $\left(F_{1,224.6}=\right.$ $3.607, p=0.060)$, though it increased as a function of per cent emotion in the morph $\left(F_{1,201.1}=6.330\right.$, $p=0.012) ;(2)$ the response speed increased at a rate of $0.13192 \pm 0.01349 \mathrm{~s}$ every $10 \%$ increment in

673 the morph $\left(F_{1,195}=95.637, p<0.001\right)$, independently from other experimental factors like 674 action/emotion Congruency $\left(F_{1,195}=0.038, p=0.84\right)$, Action Ordering $\left(F_{1,39}=0.98, p=0.32\right)$ and 675 Target Emotion $\left(F_{1,39}=1.45, p=0.235\right)$. The lack of interaction between OT (i.e., response time) 676 and experimental factors supports our decision to focus the following analysis on indices of signal 677 detection performance.

\section{Results and discussion}

\section{Statistical analyses}

680 Following Knoblauch and Maloney (2012), all indices of signal detection performance (both 681 perceptual and decision based) were calculated by applying a generalized linear model $(\mathrm{glm})$ with a 
682 probit link function to the whole set of binary responses. Individual triplets of $d$ ' values associated to 683 the three combinations of hits (yes responses to 10,20,30\% morph, respectively) and false alarms 684 (yes responses to $0 \%$ morph) were extracted using a $g l m$ with variable intercept $\beta_{1}$ and slope $\beta_{2}$ for 685 every participant, reaching session, emotion, and action ordering. We encoded signal 686 presence/absence as a discrete variable ( 1 for trials with morph intensity $>0$; 0 for trials with the 687 original neutral face) and reparametrized each individual Gaussian function fit in term of its slope (corresponding to the difference between hit and false alarm rates on the probit scale), or $d^{\prime}$.

Generalizing upon this statistical technique and following Marneweck, Loftus, and Hammond (2013) we further extracted two global indices of detection performance by fitting the same $\mathrm{glm}$ to the entire set of yes responses as a function of signal presence/absence. The two indices were global $d^{\prime}$ for the perceptual component of the model and response criterion $c$ [as given by $-\frac{\left(2 \beta_{1}+\beta_{2}\right)}{2}$ ] for the decision component. The $c$ index provides a measure of response bias independent of sensitivity to facial expressions of emotion, as needed to draw conclusions relevant to our second goal (G2). Individual $c$ values indicate how far the criterion used by the observer to deliver a yes response departs from the optimal decision rule (i.e., equal false alarm and miss rates), with negative $c$ values indicating an unbalance in favor of yes over no responses. On the average, a negative $c$ value was expected as a consequence of the unbalanced $[\mathrm{S}+\mathrm{N}] /[\mathrm{N}]$ ratio (with only $1 / 4$ of trials displaying a neutral face).

To provide an additional measure of a possible mood-congruent effect on sensitivity to facial emotions, revealed by our detection task, we also analyzed $A T$ values as derived from intercept and slope values estimated by a linear mixed-effect (lme) model with participants as a random effect and

703 704

705

706

707

708

709

710

711 morph intensity as the continuous covariate of individual $d^{\prime}$ triplets applied to each of the 4 experimental groups of participants separately.

The negative sign of the slope between individual $d^{\prime}$ triplets and per cent target emotion was used as exclusion criterion, given that $A T$ values computed from an indirect relationship between $d^{\prime}$ and morph intensity were statistically meaningless: this led to the removal of one participant from the analysis of data from Experiment 1 (out of the total of 40).

Distributions of individual values of performance indices $d^{\prime}$, global $d^{\prime}, A T, c$ were analyzed using a step-wise procedure that contrasted linear mixed-effect (lme) models of increasing complexity (Bates \& Machler, 2014), depending on the number of fixed effects, modelled by the 
712 factors of our experimental design (action/emotion valence Congruency, Target Emotion, and Action

713 Ordering). Models were fitted using Restricted Maximum Likelihood. Participants were treated as

714 random effects so to control for the individual variability of emotion detection performance. We

715 followed Bates (2010) and used this statistical procedure to obtain two-tailed $p$-values by means of

716 likelihood ratio test based on $\chi^{2}$ statistics when contrasting lme with different complexities (for a

717 discussion of advantages of a lme procedure over the more traditional mixed models analysis of

718 variance see Kliegl, Wei, Dambacher, Yan \& Zhou, 2010). We used type 3 like two tailed $p$-values

719 for significance estimates of $l m e$ 's fixed effects and parameters adjusting for the $F$-tests the

720 denominator degrees-of-freedom with the Satterthwaite approximation based on SAS proc mixed

721 theory. Among the indices that have been proposed as reliable measures of the predictive power and

722 of the goodness of fit for lme models we selected the concordance correlation coefficient $r c$, which

723 provides a measure of the degree of agreement between observed and predicted values in the $[-1,1]$

724 range (Vonesh, Chinchilli \& Pu, 1996; Rigutti, Fantoni, Gerbino, 2015). Post-hoc tests were

725 performed using paired two sample t-tests with equal variance. As measures of significant effect

726 size, depending on the statistical analysis, we provided Cohen's $d$, the coefficient of determination $r^{2}$,

727 and/or $r_{c}$.

\section{Evidence from the distributions of yes responses}

Fig. 4 illustrates the average percentages of yes ("Emotion present") responses (and SEMs)

730 together with the best fitting cumulative Gaussian (averaged across participants as modelled through

$731 \mathrm{glm}$ ) as a function of per cent emotion for the two levels of action/emotion Congruency: congruent

732 (red) vs. incongruent (cyan). Panels A,C show detection data from Experiments 1 and 3, following

733 uncomfortable-comfortable MAMIP sessions; panels B,D show detection data from Experiments 2

734 and 4, following comfortable-uncomfortable MAMIP sessions. Emotions to be detected were

735 happiness for panels A,B and anger for panels C,D. 


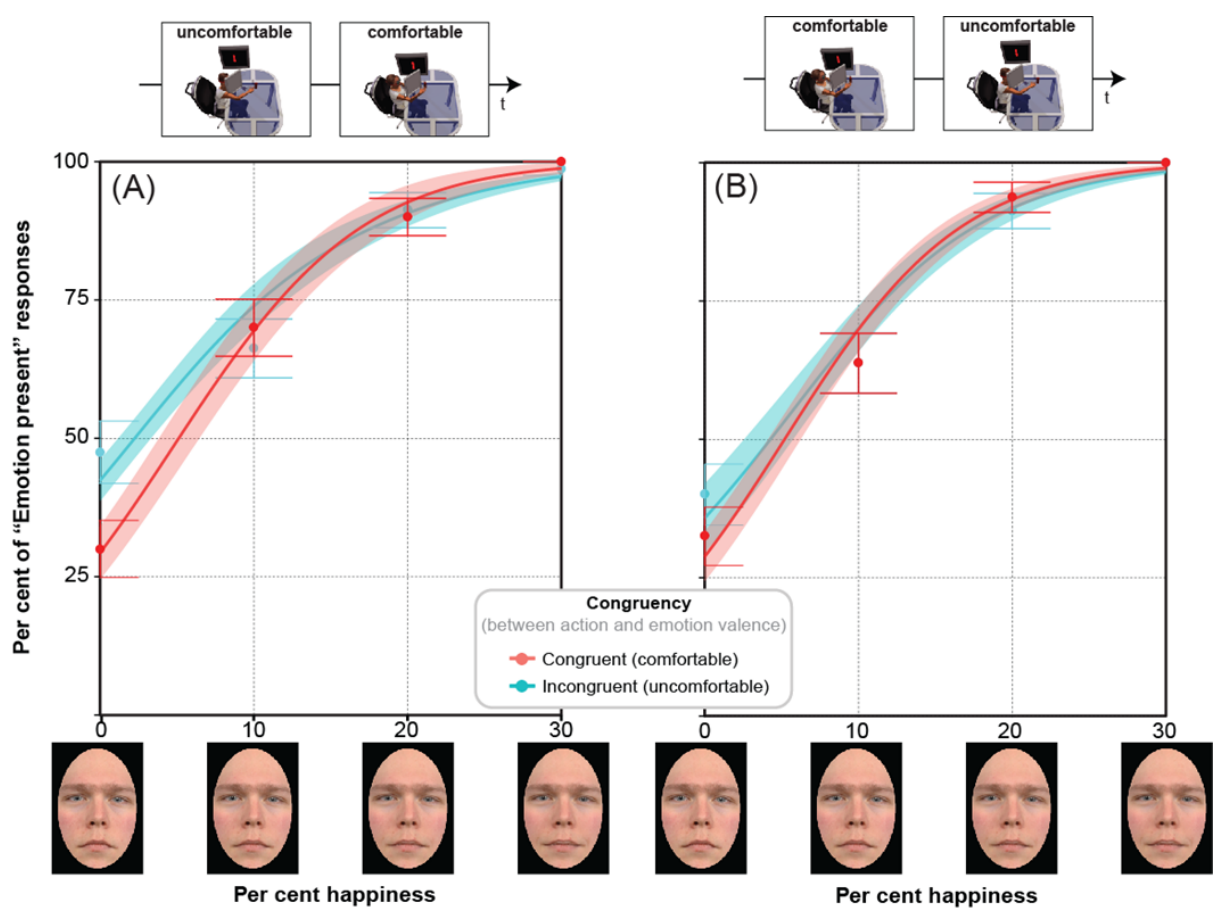

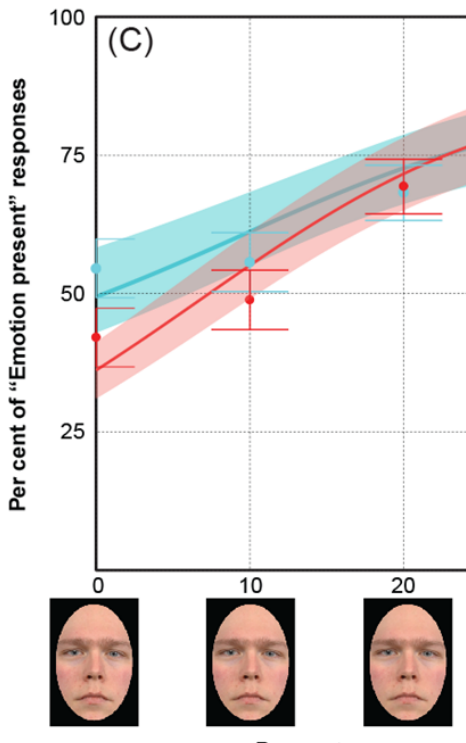

Per cent anger

Per cent happiness

Figure 4. Modeling the distributions of "Emotion present" percentages with $\mathbf{g l m}$. The four panels show the average percentages of yes ("Emotion present") responses \pm SEM as a function of per cent emotion, when the target emotion was congruent/incongruent (red/cyan symbols, respectively) with action/mood valence. Red/cyan curves are the best average cumulative Gaussian fits of response percentages, with shaded bands indicating \pm standard error of regression. Action ordering is illustrated by the legend on top. Top panels A,B refer to happiness detection (A: Experiment 1, uncomfortable $\Rightarrow$ comfortable; B: Experiment 2, comfortable $\Rightarrow$ uncomfortable); bottom panels C,D refer to anger detection (C: Experiment 3, uncomfortable $\Rightarrow$ comfortable; D: Experiment 4, comfortable $\Rightarrow$ uncomfortable).

A preliminary statistical analysis revealed that the $\mathrm{glm}$ procedure used to extract our indices of detection performance provided a very good fit to our dataset and was robust enough to describe the 
748 best linear fit describing the relationship between individual predicted and individual observed yes percentages was a line with unitary slope and null intercept accounting for a large percentage of variance, as shown in Table 1.

751

Table 1. Summary table of yes distributions in Experiments 1-4. Per cent explained variance and statistical indices of goodness of fit of glm-based vs. observed yes distributions in Experiments 1-4.

\begin{tabular}{|c|c|c|c|c|}
\hline & \multirow[b]{2}{*}{$\begin{array}{l}\text { Per cent } \\
\text { explained } \\
\text { variance }\end{array}$} & \multirow[b]{2}{*}{ Goodness of Fit } & \multicolumn{2}{|c|}{ best $g / m$ fitting parameters } \\
\hline & & & $\beta 1$ & $\beta 2$ \\
\hline $\begin{array}{l}\text { Experiment } 1 \\
\text { (uncomfortable } \\
\Rightarrow \text { comfortable) }\end{array}$ & 86 & $\begin{array}{c}\mathrm{F}(1,78)=504.0 \\
p<0.001\end{array}$ & $\begin{array}{c}0.002 \pm 0.035 \\
t=-0.059, p=0.95\end{array}$ & $\begin{array}{c}1.00 \pm 0.045 \\
\mathrm{t} \text { vs. } 1=0.02, p=0.98\end{array}$ \\
\hline $\begin{array}{c}\text { Experiment } 2 \\
\quad \text { (comfortable } \\
\Rightarrow \text { uncomfortable) }\end{array}$ & 88 & $\begin{array}{c}\mathrm{F}(1.78)=595.7 \\
p<0.001\end{array}$ & $\begin{array}{c}0.005 \pm 0.031 \\
t=0.17, p=0.86\end{array}$ & $\begin{array}{c}0.99 \pm 0.040 \\
\mathrm{t} \text { vs. } 1=0.18, p=0.85\end{array}$ \\
\hline $\begin{array}{l}\text { Experiment } 3 \\
\text { (uncomfortable } \\
\Rightarrow \text { comfortable) } \\
\end{array}$ & 86 & $\begin{array}{c}\mathrm{F}(1,78)=551.9 \\
p<0.001\end{array}$ & $\begin{array}{c}0.033 \pm 0.030 \\
t=-1.07, p=0.28\end{array}$ & $\begin{array}{c}1.05 \pm 0.044 \\
\mathrm{t} \text { vs. } 1=0.77, p=0.44\end{array}$ \\
\hline $\begin{array}{c}\text { Experiment } 4 \\
\quad \text { (comfortable } \\
\Rightarrow \text { uncomfortable) }\end{array}$ & 73 & $\begin{array}{c}\mathrm{F}(1,70)=185.2 \\
p<0.001\end{array}$ & $\begin{array}{c}0.001 \pm 0.05 \\
t=0.17, p=0.87\end{array}$ & $\begin{array}{l}0.98 \pm 0.070 \\
\text { t vs. } 1=0.15, p=0.87\end{array}$ \\
\hline
\end{tabular}

The graphs in Fig. 4 clearly show the effect of action/emotion congruency on sensitivity. The pattern of responses is fully consistent with the facilitation-by-congruency hypothesis and at odds with both a facilitation-by-arousal hypothesis and explanations based on action valence per se. In all panels the increase of yes responses as a function of per cent emotion is well described by two positive halves of a sigmoid, with the red curve fitting the data from the block in which the valence of the target emotion was congruent with the valence of the reaches (Fig. 4A, 4B: happiness detection after comfortable reaches; Fig 4C, 4D: anger detection after uncomfortable reaches) vs. the cyan curve fitting the data from the block in which the valence of the target emotion was incongruent with the valence of the reaches (panels A, B: happiness detection after uncomfortable reaches; panels C,D: anger detection after comfortable reaches).

Consistently with the facilitation-by-congruency hypothesis, yes percentages monotonically increased with morph intensity, with the rate of increase being larger (indicating higher sensitivity) for the red mood-congruent curve than the cyan mood-incongruent one, in all tested conditions: the yes percentage was indeed smaller in the mood-congruent (red points) than mood-incongruent (cyan points) conditions for small (determining an overall lower false alarm rate) but not large values of 
771 per cent emotion in the morph (determining an overall higher hit rate), in both anger and happiness

772 detection tasks. This was confirmed by the results of the lme analysis revealing that the pattern of 773 average "happy" percentages $\left(t=31.7, d f=158 ; p=0.00 ; r^{2}=0.86,95 \%\right.$ CI [0.82, 0.90], $r_{c}=0.93$, $77495 \%$ CI $[0.90,0.95])$ determined a significant main effect of Morph Intensity $\left(F_{3,140}=278.4, p<\right.$ $7750.001)$, and a significant Morph Intensity $\times$ Congruency interaction $\left(F_{3,140}=4.078, p=0.008\right)$. The 776 pattern of average "angry" percentages was similarly distributed $\left(t=31.7, d f=158 ; p=0.00 ; r^{2}=\right.$ $7770.80,95 \%$ CI $[0.74,0.85], r_{c}=0.88,95 \%$ CI $\left.[0.85,0.91]\right)$, though not determining a fully significant

778 Morph Intensity $\times$ Congruency interaction $\left(F_{3,133}=2.10, p=0.10\right)$, probably because of the overall 779 noisier pattern of responses: the consistency of "angry" percentages (average individual variability of 780 yes percentages quantified by average standard error of the mean values) being worse in the anger 781 (0.0563) than in the happiness (0.035) detection task $(t=2.867, d f=30, p=0.007, d=1.04)$. The 782 interaction patterns rising from both distributions of yes percentages were due to the negative 783 congruency gain (Per cent yes congruent - Per cent yes incongruent) at 0\% emotion (Experiments 1 784 and 2, Fig. 4A,B: $-0.125 \pm 0.034, t=-3.649, d f=140, p<0.001$; Experiments 3 and 4, Fig. 4C,D: $7850.088 \pm 0.040, t=-2.221, d f=133, p=0.028)$, combined with a positive or null gain for positive at $786 \quad 10-30 \%$ emotion.

787 Notably, the higher sensitivity for detecting happiness after comfortable rather than 788 uncomfortable reaches (Experiments 1 and 2, Fig. 4A,B) is consistent with facilitation-by789 congruency but not facilitation-by-arousal, given that an arousal-based improvement in emotional 790 face processing should occur after the uncomfortable MAMIP session (requiring a higher level of 791 motor activation/arousal than the comfortable). Furthermore, the pattern of yes responses depicted in 792 Fig. 4 rules out any explanation based on action valence per se. Reaches of opposite valence led to 793 similar improvements of emotion detection performance, consistently with action/emotion valence 794 congruency: in Experiments 1 and 2 (Fig. 4A,B) happiness detection was improved after a 795 comfortable (not uncomfortable) MAMIP session; while in Experiments 3 and 4 (Fig. 4C,D) anger 796 detection was improved after an uncomfortable (not comfortable) MAMIP session. Confirming 797 previous results (Fantoni \& Gerbino, 2014), detection of facial emotions was improved by the 798 congruency between the valence of the inducing actions and the valence of the target emotion. 
799 Evidence from the distributions of sensitivities, thresholds and response 800 bias

801 Conclusions from the previous analysis on yes percentages were corroborated by lme statistics 802 on indices of detection performance. These quantitative analyses also allowed us answering two 803 major questions about the facilitation-by-congruency effect induced by MAMIP: (1) is it an additive 804 or multiplicative effect?; (2) is it a perceptual or post-perceptual effect?

805 Action/emotion valence congruency improves happiness detection but not response 806 bias: against arousal

$807 \quad$ Fig. 5 shows the average $d^{\prime}$ triplets (panels A,E), $A T$ (panels B,C,F,G) and $c$ (panels D,H) for

808 the two reaching sessions in Experiments 1 (panels A,B,C,D, uncomfortable $\Rightarrow$ comfortable) and 2 809 (panels E,F,G,H, comfortable $\Rightarrow$ uncomfortable). Independent of Action Ordering (panels A vs. E)

810 the distributions of average $d$ ' values for happiness detection as a function of per cent target emotion

811 were in strong agreement with an additive (not multiplicative) facilitation-by-congruency effect, as

812 confirmed by the lme analyses of Experiments 1 and 2, with participants as random effects, and

813 emotion Congruency (comfortable action $\Rightarrow$ happy expression vs. uncomfortable action $\Rightarrow$ happy

814 expression) and Morph Intensity (10, 20, 30\% emotion) as fixed effects. A separate analysis of data

815 from Experiments 1 and 2 follows.

816 In Experiment 1 (Fig. 5A), happiness detection increased linearly with an lme estimated rate of 817 about $2.6 \pm 0.26 d^{\prime}$ units every 10 per cent increment in the morph $\left(F_{1,48}=138.56, p<0.001\right)$, and 818 with a constant $d$ ' increment of about $0.84 \pm 0.36$ units in congruent (comfortable-happy) over 819 incongruent (uncomfortable-happy) conditions $\left(F_{1,48}=5.49, p=0.023\right)$. Only 50 reaching acts 820 distributed over $10 \mathrm{~min}$, with a slightly different depth extent (average depth difference between 821 comfortable and uncomfortable reaches $=17.56 \mathrm{~cm} \pm 0.18$ ), produced systematic changes in the 822 detection of subtle variations of happiness. This is consistent with the idea that reaches with positive 823 (i.e., comfortable) but not negative (i.e., uncomfortable) valence pre-activate detectors selectively 824 tuned to emotional facial features with the same valence (i.e., happiness), which in turn facilitate the 825 performance in Congruent relative to Incongruent conditions. 
(A)

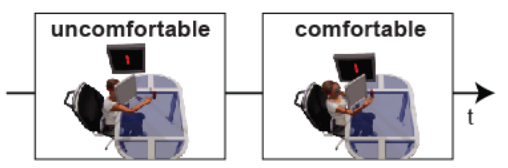

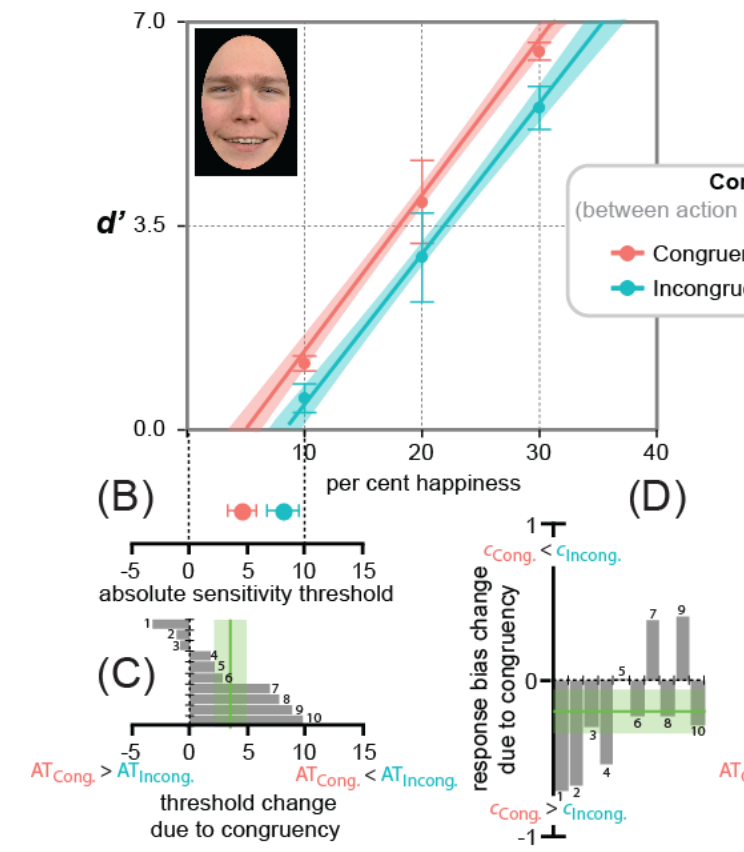

(E)
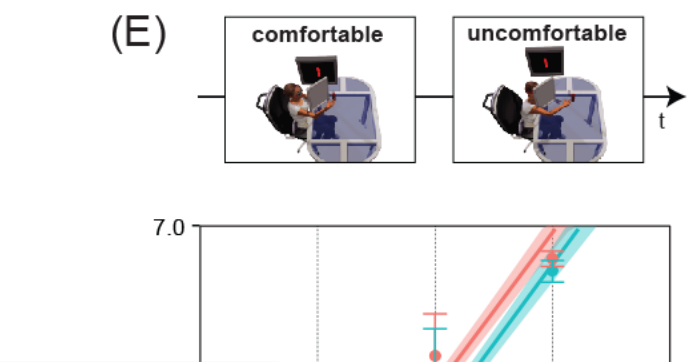

Congruency

ortable)

uent (uncomfortable)
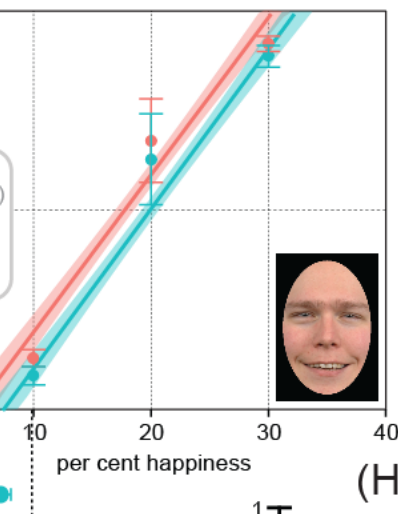

(F)
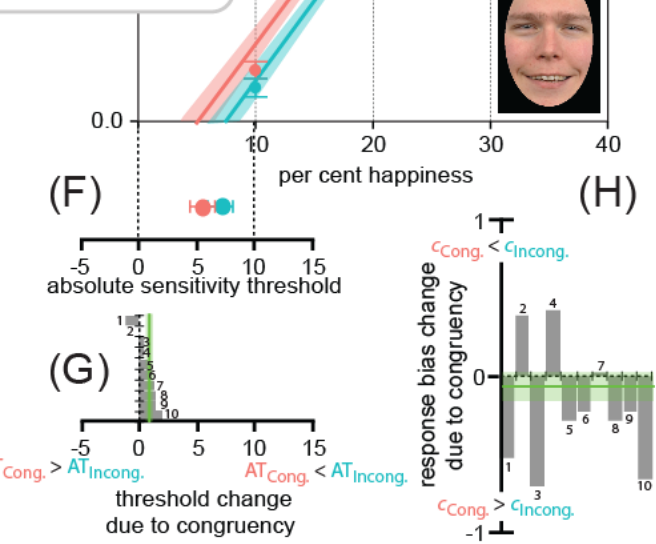

Figure 5. Action/emotion valence congruency affects happiness detection sensitivity, but not response bias. Average $d^{\prime}$ triplets (A, E) and average $A T \mathrm{~s}(\mathrm{~B}, \mathrm{~F})$, for the congruent (comfortable, in red) and incongruent (uncomfortable, in cyan) reaching sessions $(\mathrm{B}, \mathrm{F})$, together with individual $A T \mathrm{~s}(\mathrm{C}, \mathrm{G})$ and response biases $(\mathrm{D}, \mathrm{H})$ changes due to congruency, in Experiments 1 (uncomfortable $\Rightarrow$ comfortable) and 2 (comfortable $\Rightarrow$ uncomfortable) as coded by the icons on top. Error bars represent \pm SEM. Red and cyan lines in panels A, E are the lme model regression line, with the shaded region corresponding to \pm standard error of the regression. The spatial arrangement of these lines in the $d^{\prime}$ by per cent emotion graph is informative about the influence of action/emotion valence congruency on happiness detection, with a facilitation-by-congruency effect signaled by the red line being above the cyan line and with their parallelism being diagnostic of an additive (not multiplicative) effect. (B, F) A red point closer to zero than the cyan indicates a lowering of the absolute threshold for happiness induced by congruency. (C, G) Individual threshold changes between incongruent and congruent reaching sessions in Experiments 1 and 2, respectively. A negative change represents an increased likelihood that a smaller value of per cent happiness in the morph would elicit a yes response after the incongruent than congruent session. The vertical green line represents the grand average threshold change due to congruency \pm SEM. (D, H) Individual response bias changes between incongruent and congruent reaching sessions in Experiments 1 and 2, respectively. A negative change represents an increased likelihood towards a positive bias after the congruent than incongruent session. The vertical green line represents the grand average response bias change due to congruency \pm SEM.

844

Remarkably, no improvement of fit was found $\left(\chi^{2}{ }_{1}=0.18, p=0.67\right)$ with a separate slope lme

including the Morph Intensity $\times$ Congruency interaction $\left(F_{3,56}=43.44, p<0.001, r^{2}=0.74, r_{c}=0.85\right.$, 
851 This is corroborated by the post-hoc analyses (one tailed paired $t$ test) showing that any $10 \%$ 852 increment in morph intensity (i.e., from 10 to 20 or from 20 to 30) produced a significant $d^{\prime}$ gain (see 853 Table 2). These $d^{\prime}$ gains were similar in magnitude in incongruent-uncomfortable $(t=0.78, d f=9, p$ $854=0.45, d=0.52)$ and congruent-comfortable conditions $(t=0.78, d f=9, p=0.45, d=0.52)$.

Table 2. Summary of post hoc analyses for Experiments 1 and 2.

\begin{tabular}{|c|c|c|c|c|}
\hline & & & \\
\hline & & $\begin{array}{c}\text { per cent } \\
\text { happiness range }\end{array}$ & $\begin{array}{c}\text { mean } d^{\prime} \text { gain and } \\
\text { SEM }\end{array}$ & paired $t$ test \\
\hline \multirow{4}{*}{ Experiment 1} & \multirow{2}{*}{ Incongruent } & [10\%-20\%] & $2.41 \pm 0.65$ & $\begin{array}{c}t=3.80, d f=9 \\
p=0.004, d=2.53\end{array}$ \\
\hline & & [20\%-30\%] & $2.57 \pm 0.73$ & $\begin{array}{c}t=3.94, d f=9 \\
p=0.003, d=2.62\end{array}$ \\
\hline & \multirow{2}{*}{ Congruent } & [10\%-20\%] & $2.77 \pm 0.75$ & $\begin{array}{c}t=4.11, d f=9 \\
p=0.003, d=2.76\end{array}$ \\
\hline & & [20\%-30\%] & $2.58 \pm 0.81$ & $\begin{array}{c}t=3.62, d f=9 \\
p=0.005, d=2.23\end{array}$ \\
\hline \multirow{4}{*}{ Experiment 2} & \multirow{2}{*}{ Incongruent } & [10\%-20\%] & $3.37 \pm 0.82$ & $\begin{array}{c}t=4.51, d f=9 \\
p=0.001, d=3.00\end{array}$ \\
\hline & & [20\%-30\%] & $2.15 \pm 0.81$ & $\begin{array}{c}t=2.86, d f=9 \\
p=0.019, d=1.90\end{array}$ \\
\hline & \multirow{2}{*}{ Congruent } & [10\%-20\%] & $3.47 \pm 0.72$ & $\begin{array}{c}t=5.32, d f=9 \\
p<0.001, d=3.55\end{array}$ \\
\hline & & [20\%-30\%] & $2.06 \pm 0.77$ & $\begin{array}{c}t=3.09, d f=9 \\
p=0.013, d=2.06\end{array}$ \\
\hline
\end{tabular}

857

858

859

860

861

862

863

864

865

866

867

868

869

870

.The additive facilitation produced by MAMIP on happiness detection performance is further corroborated by the significant facilitation-by-congruency effect on: (1) global happiness sensitivity $\left(F_{1,9}=9.46, p=0.01, d=1.94\right)$, which was larger after the comfortable (global $\left.d^{\prime}=1.76\right)$ than uncomfortable (global $d^{\prime}=1.20$ ) reaching session, 95\% CI [0.15, 0.97]; (2) the absolute threshold for happiness $\left(F_{1,9}=5.79, p=0.03, d=-1.52\right)$, given that, as shown in Fig. 5C, the $A T$ value was smaller after participants completed a comfortable $(A T=4.6 \pm 1.2 \%)$ than uncomfortable $(8.1 \pm$ $1.4 \%$ ) MAMIP session, 95\% CI [0.21, 6.77].

As shown in Fig. 5D, the response bias was clearly influenced by the unbalanced $[\mathrm{S}+\mathrm{N}] /[\mathrm{N}]$ trial ratio. As expected, the 3:1 ratio produced an overall negative bias; i.e., a dominance of "Emotion present" over "Emotion absent" responses in both Congruent $(-0.31 \pm 0.13, t=-2.714, d f=$ $15.04, p=0.016, d=-1.40)$ and Incongruent conditions $(-0.52 \pm 0.10, t=-4.603, d f=15.04, p<$ 0.001, $d=-2.37$ ). However, unlike sensitivity, the bias was independent of action valence (comfortable vs. uncomfortable). To control for the perceptual vs. post-perceptual locus of the 
871 MAMIP effect, according to $G 2$, the same lme analysis conducted on perceptual indices of happiness 872 detection (global d' and $A T$ ) was conducted on response criterion $c$. The MAMIP effect was not 873 replicated. The main effect of Congruency on $c$ was non significant $\left(F_{1,9}=3.0, p=0.12\right)$.

874 As depicted in Fig. 5E,F,G, and H, the facilitation-by-congruency effect was strikingly similar 875 in Experiment 2 (uncomfortable $\Rightarrow$ comfortable). Again, the pattern of happiness detection 876 performance shown in Fig. 5E was optimally accounted for by an equal slope $\operatorname{lme}\left(F_{2,57}=71.85, p<\right.$ $8770.001, r^{2}=0.79, r_{c}=0.88,95 \%$ CI $\left.[0.81,0.92]\right)$, consistent with an additive facilitation-by878 congruency effect induced by MAMIP, not by a separate slope lme $\left(F_{3,56}=47.06, p<0.001, r^{2}=\right.$ $\left.8790.79, r_{c}=0.88,95 \% \mathrm{CI}[0.81,0.92]\right)$, consistent with a multiplicative facilitation by congruency 880 effect induced by MAMIP $\left(\chi^{2}{ }_{1}=0.0, p=0.99\right)$. The lme analysis revealed a similar, though not 881 significant $\left(F_{1,48}=1.46, p=0.23\right)$, constant increment of sensitivity due to action/emotion valence 882 congruency $(0.23 \pm 0.30)$, and a similar linear modulation of sensitivity by the per cent happiness in 883 the morph $\left(\beta_{2}=2.76 \pm 0.20, F_{1,48}=179.96, p<0.001\right)$. This was confirmed by post-hoc paired $t$ 884 tests. As in Experiment 1, any 10 per cent increment in morph intensity (i.e., from 10 to 20 or from 88520 to 30) produced a significant $d^{\prime}$ gain (see Table 2), with the $d^{\prime}$ gains being similar in magnitude in 886 incongruent-uncomfortable $(t=0.78, d f=9, p=0.45, d=0.52)$ and congruent-comfortable 887 conditions ( $t=0.78, d f=9, p=0.45, d=0.52$ ). Finally the average difference between $d^{\prime}$ values for 888 congruent and incongruent conditions was almost constant at increasing per cent happiness in the 889 morph, with the performance gain due to congruency for 10,20, and 30 per cent happiness in the 890 morph being equal to: $0.20 \pm 0.14(t=1.81, d f=9, p=0.09, d=1.20), 0.29 \pm 0.087 \quad(t=3.83, d f=$ $8919, p=0.004, d=2.55)$, and $0.20 \pm 0.088(t=2.68, d f=9, p=0.025, d=2.68)$, respectively.

892 The different facilitation-by-congruency effect sizes revealed by the lme analyses in 893 Experiments 1 and 2 were likely due to the unbalanced temporal ordering of reaching sessions.

894 Participants in Experiment 2 were indeed less experienced with both the detection task and the face 895 set after the comfortable (congruent) than uncomfortable (incongruent) MAMIP session, given that 896 the comfortable condition occurred first.

897 Surprisingly, despite the fact that the effects of action/emotion valence congruency and 898 learning were in opposite directions in Experiment 2, thus reducing the performance difference 899 induced by the two reaching sessions, we found the same significant facilitation-by-congruency 900 effect observed in Experiment 1 on both global d' $\left(F_{1,9}=8.09, p=0.02, d=1.79\right)$ and $A T\left(F_{1,9}=\right.$ $9018.45, p=0.02, d=1.84)$, even in the absence of significant shifts in response bias $c\left(F_{1,9}=0.96, p=\right.$ 
902 0.37). As shown in Fig. 5F,G, action/emotion valence congruency increased global d' by about 0.24 $903 d^{\prime}$ units, 95\% CI [0.05, 0.43], and decreased $A T$ by about 0.83 per cent emotion, 95\% CI [0.05, 0.43]. 904 Again, similar, though negative, $c$ values were obtained in congruent $(-0.32 \pm 0.10, t=-2.85, d f=$ $90511.52, p=0.015, d=-1.68)$ and incongruent $(-0.40 \pm 0.12, t=-3.53, d f=15.04, p=0.004, d=-2.10)$ 906 conditions (Fig. 5H), with an average response bias change due to congruency of about $-0.077,95 \%$ 907 CI $[-0.26,0.10])$.

908 909

910

911

912

913 914

915

916

917

918

919

920

921

922

923

924

925

926

927

928

929

930

931

\section{Is there an effect of Action Ordering on happiness detection?}

To test the possible role of Action Ordering we compared the patterns of $d^{\prime}, A T$ and $c$ in Experiment 2 directly to those of Experiment 1, including Experiment as an additional fixed effect in lme analyses.

In a first lme analysis we thus asked how the relationship between individual $d$ ' values and morph intensities were affected by Congruency and/or Action Ordering. In this model $(t=19.66, d f$ $=118, p=0.00, r^{2}=0.7795 \%$ CI $[0.68,0.83], r c=0.86,95 \%$ CI $\left.[0.81,0.90]\right) d$ ' resulted to be positively affected by Morph Intensity $\left(\beta_{2}=0.27 \pm 0.015, F_{1,98}=315.19, p<0.001\right)$ and Congruency (estimated $d^{\prime}$ gain $=0.53 \pm 0.24, F_{1,98}=4.73, p=0.03$ ): other main effects or interactions were not statistically significant $\left(\chi_{5}^{2}=3.08, p=0.68\right)$, with the intercept of the equal slope lme model, for the mood congruent condition, being negative $\left(\beta_{1}=-1.49 \pm 0.37, d f=113.56, t=-4.02, p<0.001, d=\right.$ 0.754). A second lme model on global d' $\left(t=10.60, d f=38, p=0.00, r^{2}=0.75,95 \%\right.$ CI [0.57, 0.83], $r c=0.79,95 \%$ CI $[0.68,0.86])$ revealed a significant main effect of facilitation by congruency of about $0.4 \pm 0.1 d^{\prime}$ units $\left(F_{1,18}=15.94, p<0.001\right)$; while neither the effect of Experiment $\left(F_{1,18}=\right.$ $0.0020, p=0.96)$ nor the Congruency $\times$ Experiment interaction $\left(F_{1,18}=2.49, p=0.13\right)$ were significant. Similar results were obtained on $A T \mathrm{~s}$, given that facilitation-by-congruency, again, resulted to be the only significant factor affecting the performance (estimated $A T$ after comfortablecongruent $=5.24 \pm 0.82$ per cent emotion; estimated $A T$ after uncomfortable-incongruent $=7.40 \pm$ 0.84 per cent emotion; $\left.F_{1,18}=8.51, p=0.001\right)$.

\section{Does MAMIP affect response bias in happiness detection?}

In a final lme analysis we asked whether these effects occur at the level of response or stimulus encoding. Following Signal Detection Theory (Macmillan \& Creelman, 2004) a pure perceptual effect is supported by the full independence between results on sensitivity and response bias. Specifically the effect of congruency measured on the perceptual indices of performance ( $d$ ' and $A T$ ), 
932 should result to be absent when measured on the decision index of performance (c). This is what we

933 found, as an lme model on $c$ revealed no significant main or interaction effects: no significant

934 decrement of fit was indeed found when contrasting a full lme model including Congruency,

935 Experiment, and their interaction as fixed effects with a baseline lme model with no fixed effects $\left(r_{c}\right.$

936 slightly decreases from $0.83,95 \%$ CI $[0.74,0.89]$ to $0.76,95 \%$ CI $[0.65,0.84] ; \chi^{2}{ }_{3}=4.8, p=0.18$ ).

937 In the present investigation, therefore, there is no evidence that learning and arousal contribute

938 to the perceptual response beyond what emotion intensity and the congruency between action and 939 emotion valence can explain.

940

941

942

943

944

945

946

947

948

950

951

952

953

954

955

956

957

958

959

960

961

\section{Action/emotion valence congruency improves anger detection but does not modify response bias: against valence per se}

The results of Experiments 1 and 2 corroborated the idea that the facilitation-by-congruency effect induced by MAMIP was additive, robust, independent of learning and arousal, and perceptually-based: the processing of positive emotional features gets more salient after comfortable (congruent) then comfortable (incongruent) reaches. However, the result cannot be generalized to the entire affect domain, being specific for positive emotions (i.e., happiness), and cannot be interpreted univocally given that, in principle, it might have been produced by action valence per se: with happiness detection being facilitated by a comfortable action sequence, regardless of the correspondence between the valence of the mood induced by the action sequence and the emotion to be detected. If the MAMIP effect is general and independent from action valence then anger detection performance in Experiments 3 and 4 should be facilitated by uncomfortable actions, thus producing an action/emotion congruency effect similar to the one observed in Experiments 1 and 2.

This expectation closely matches results of Experiments 3 (uncomfortable $\Rightarrow$ comfortable) and 4 (comfortable $\Rightarrow$ uncomfortable) shown in Fig. 6. The distributions of average $d$ ' resulting from each unique combination of morph intensity $(10,20,30 \%)$, emotion and reaching sessions shown in Fig. 6A,E are in strong agreement with an additive (not multiplicative) facilitation-bycongruency effect: in both action ordering conditions (uncomfortable $\Rightarrow$ comfortable in Fig. 6A; comfortable $\Rightarrow$ uncomfortable in Fig. 6E) $d^{\prime}$ increases linearly as a function of per cent anger in the morph (coded on the $x$-axis), and is aided by congruency (coded by colors with congruentuncomfortable in red, and incongruent-comfortable in cyan) by an almost constant amount at increasing per cent anger in the morph values. 
(A)
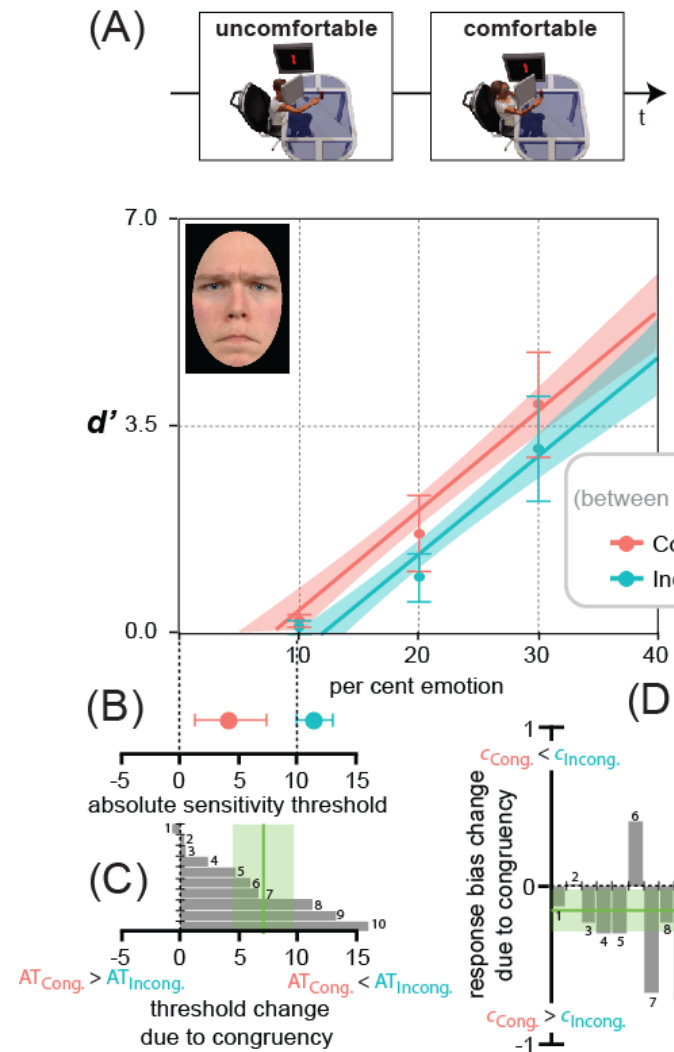

(E)
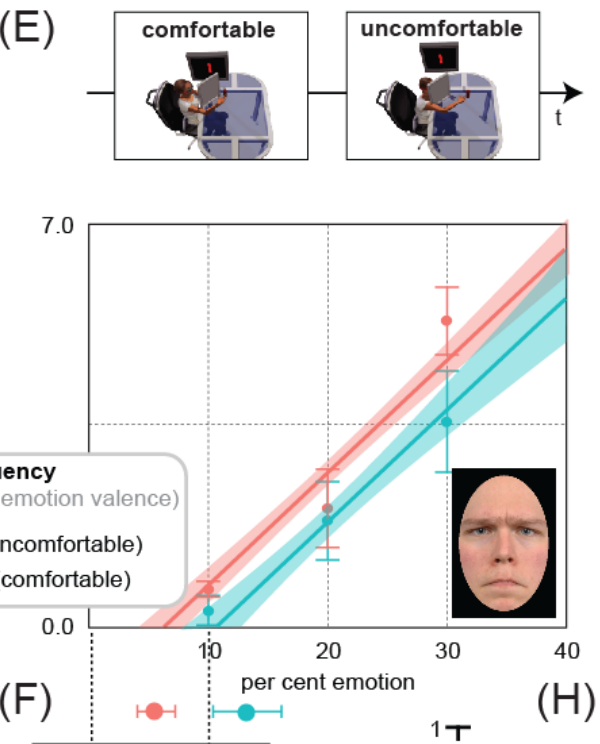

(H)
963

964

965

966

967

968

969

970

971

972

973

974

975

976

977

978

979

980

981

982

983

984

Figure 6. Action/emotion valence congruency affects anger detection, but does not modify response bias. Average $d^{\prime}$ triplets (A, E) and average $A T \mathrm{~s}$, for the congruent (uncomfortable, in red) and incongruent (comfortable, in cyan) reaching sessions $(\mathrm{B}, \mathrm{F})$, together with individual $A T \mathrm{~s}(\mathrm{C}, \mathrm{G})$ and response biases $(\mathrm{D}, \mathrm{H})$ changes due to congruency, in Experiment 3 (uncomfortable $\Rightarrow$ comfortable) and 4 (comfortable $\Rightarrow$ uncomfortable), as coded by the icons on top. Error bars represent \pm SEM. The cyan and red lines in $(\mathrm{A}, \mathrm{E})$ are the lme model regression line and the shaded region corresponds to \pm standard error of the regression (same interpretation as in Fig. 5). (B, F) A red point closer to zero than the cyan point indicates an absolute anger sensitivity threshold decrement induced by congruency. (C, G) Individual threshold changed between the incongruent and congruent reaching sessions in Experiments 1 and 2, respectively (same axis encoding as in Fig. 5). The vertical green line represents the grand average threshold change due to congruency \pm SEM. (D, H) Individual response bias changed between the incongruent and congruent reaching sessions in Experiments 1 and 2, respectively (same axis encoding as in Fig. 5). The vertical green line represents the grand average response bias change due to congruency \pm SEM.

This observation was confirmed by an lme analysis testing the effects of Morph Intensity, Action Ordering, and action/emotion Congruency on $d^{\prime}$ triplets. The analysis revealed that Morph Intensity $\left(\beta_{2}=0.181 \pm 0.0175 ; \mathrm{F}_{1,93}=107.25, p=0.00\right)$, and action/emotion Congruency (estimated $d^{\prime}$ gain due to congruency $=0.648 \pm 0.29 ; \mathrm{F}_{1,93}=5.14, p=0.024$ ) were the only factors affecting anger sensitivity $\left(t=14.30, d f=112, p=0.00, r^{2}=0.64,95 \% \mathrm{CI}[0.53,0.74], r c=0.77,95 \% \mathrm{CI}\right.$ $[0.69,0.83])$. No further main effects or interactions were observed $\left(\chi^{2}=4.74, p=0.45\right)$. This equal slope lme model best fitting our data set had a negative intercept in the mood congruent condition $\left(\beta_{1}=-1.35 \pm 0.47 ; d f=88.25, t=-2.90, p=0.005, d=0.64\right)$. As shown in panels A,E of Fig. 6 , anger detection sensitivities increased linearly with a similar lme estimated rate in Experiments 3 and 4 of 
985 about $1.67 \pm 0.24$ (Fig. 6A) and $1.98 \pm 0.35$ (Fig. 6E) respectively, every 10 per cent increment in the 986 neutral-to-angry morph continuum $\left(F_{2,85}=0.401, p=0.67\right)$, with an average $d^{\prime}$ gain due to 987 congruency that remained constant at increasing morph intensities in a direction consistent with an 988 additive facilitation-by-congruency hypothesis in both experiments $\left(F_{1,85}=0.174, p=0.677\right)$ : the 989 estimated $d$ ' gain due to congruency measuring about $0.77 \pm 0.31$ units in Experiment 3 (Fig. 6A, $t$ 990 vs $0=2.48, d f=8, p=0.037, d=1.76$ ) and $0.58 \pm 0.22$ units in Experiment 4 (Fig. $6 \mathrm{E}, t$ vs $0=2.38$, $991 d f=9, p=0.041, d=1.59$ ).

992 Table 3. Summary of post hoc analyses for Experiments 3 and 4.

\begin{tabular}{|c|c|c|c|c|}
\hline & & $\begin{array}{l}\text { per cent anger } \\
\text { range }\end{array}$ & $\begin{array}{c}\text { mean } d^{\prime} \text { gain and } \\
\text { SEM }\end{array}$ & Paired $t$ test \\
\hline \multirow{4}{*}{ Experiment 3} & \multirow{2}{*}{ Incongruent } & [10\%-20\%] & $0.83 \pm 0.44$ & $\begin{array}{c}t=2.29, d f=9 \\
p=0.048, d=1.52\end{array}$ \\
\hline & & [20\%-30\%] & $2.18 \pm 0.78$ & $\begin{array}{c}t=3.21, d f=9 \\
p=0.011, d=2.14\end{array}$ \\
\hline & \multirow{2}{*}{ Congruent } & [10\%-20\%] & $1.47 \pm 0.65$ & $\begin{array}{c}t=2.69, d f=9 \\
p=0.025, d=1.79\end{array}$ \\
\hline & & [20\%-30\%] & $2.17 \pm 0.86$ & $\begin{array}{c}t=2.93, d f=9 \\
p=0.017, d=1.95\end{array}$ \\
\hline \multirow{4}{*}{ Experiment 4} & \multirow{2}{*}{ Incongruent } & [10\%-20\%] & $1.55 \pm 0.75$ & $\begin{array}{c}t=2.53, d f=8 \\
p=0.035, d=1.79\end{array}$ \\
\hline & & {$[20 \%-30 \%]$} & $1.71 \pm 0.80$ & $\begin{array}{c}t=2.59, d f=8 \\
p=0.032, d=1.83\end{array}$ \\
\hline & \multirow{2}{*}{ Congruent } & [10\%-20\%] & $1.40 \pm 0.68$ & $\begin{array}{c}t=2.49, d f=8 \\
p=0.037, d=1.76\end{array}$ \\
\hline & & [20\%-30\%] & $3.25 \pm 0.79$ & $\begin{array}{c}t=4.63, d f=8 \\
p=0.002, d=3.27\end{array}$ \\
\hline
\end{tabular}

993

We further confirmed the direct increase of $d^{\prime}$ as a function of morph intensity through

995 congruent and incongruent conditions by post-hoc paired one tailed $t$-tests. As summarized in Table 3 , in Experiment 3, as anger increased from 10 to $20 \% d^{\prime}$ significantly increased by about $0.83 \pm 0.44$ $d^{\prime}$ units and $1.47 \pm 0.65 d^{\prime}$ units, after comfortable-incongruent and uncomfortable-incongruent reaches respectively; similar $d^{\prime}$ increments due to a 20 to $30 \%$ anger in the morph growth were observed after comfortable-incongruent ( $t$ vs $0.83 \pm 0.44=1.87, d f=9, p=0.093, d=1.24)$ and uncomfortable-congruent reaches $(t$ vs $1.47 \pm 0.65=1.10, d f=9, p=0.30, d=0.73)$. Post-hoc tests on $d^{\prime}$ values from Experiment 4 were strikingly similar, confirming that, independent of Action

1002 Ordering, Congruency did not affect the rate of increase of $d^{\prime}$ over morph intensity, which is 1003 consistent with a rather general additive (not multiplicative) facilitation-by-congruency effect. As by 1004 in Experiment 3, also in Experiment 4 any 10\% increment in the morph intensity (i.e., from 10 to 
$100520 \%$, or from 20 to $30 \%$ ) produced a significant $d^{\prime}$ gain (see Table 3). Furthermore, a similar $d^{\prime}$ gain 1006 was induced for 10 to $20 \%$ and a 20 to $30 \%$ anger increments in the morph by both the congruent1007 uncomfortable $(t=1.81, d f=8, p=0.11, d=1.28)$ and the incongruent-comfortable $(t=0.79, d f=8$, $1008 p=0.45, d=0.55)$ sequence of reaches.

1009 The additive facilitation produced by MAMIP on anger detection performance in both action 1010 ordering conditions produced a pattern of global sensitivity enhancement and absolute threshold 1011 decrements due to congruency that closely resembles the one observed in Experiments 1 and 2.

1012 Global d' after the uncomfortable (congruent) reaching session outperformed those after the 1013 incongruent-comfortable reaching session by: $0.24 d^{\prime}$ units, 95\% CI [-0.02, 0.50] (estimated global $1014 d^{\prime}=0.55$ and 0.79 , after comfortable and uncomfortable reaching sessions, respectively), in

1015 Experiment $3\left(F_{1,9}=4.18, p=0.05, d=1.3\right)$, and $0.35 d^{\prime}$ units, 95\% CI [-0.01, 0.71] (estimated 1016 global $d^{\prime}=0.78$ and 1.13, after comfortable and uncomfortable reaching sessions, respectively), in 1017 Experiment $4\left(F_{1,9}=8.10, p=0.02, d=1.8\right)$. These increments in sensitivity due to congruency were 1018 statistically similar across action ordering conditions as confirmed by an lme model including the 1019 Experiment as fixed effect $\left(t=13.82, d f=36, p=0.00, r^{2}=0.84,95 \%\right.$ CI [0.71, 0.91], $r_{c}=0.88$, $102095 \%$ CI $[0.81,0.93])$. The model indeed revealed a significant main effect of facilitation by 1021 congruency of about $0.29 \pm 0.09 d^{\prime}$ units $\left(F_{1,17}=9.3, p=0.007\right)$, but not of the Experiment $\left(F_{1,17}=\right.$ $10221.90, p=0.19)$, and not of the Congruency $\times$ Experiment interaction $\left(F_{1,17}=0.35, p=0.56\right)$.

1023 A similar result was obtained on individual per cent anger value above which a difference 1024 between the original neutral faces and the faces morphed with anger gets just noticeable; i.e., $A T$ 1025 (shown in Fig. 6B,F for Experiments 3 and 4 respectively). Again, the lme analysis ( $t=6.63, d f=36$, $1026 p=0.00, r^{2}=0.55,95 \%$ CI $[0.31,0.63], r_{c}=0.61,95 \%$ CI $\left.[0.45,0.74]\right)$, revealed that the only 1027 significant factor affecting $A T$ s across experiments was Congruency $\left(F_{1,17}=12.35, p=0.003\right)$, which 1028 produced an amount of $A T$ decrement that was similar, though significant, in both Experiment 3 (7.1, $102995 \%$ CI $[1.15,13.03]$ per cent anger in the morph; $F_{1,9}=7.27, p=0.02, d=1.7$, Fig. 6C) and 1030 Experiment $4\left(7.75,95 \%\right.$ CI $[0.00,15.50]$ per cent anger in the morph; $F_{1,8}=5.6, p=0.03, d=1.57$, 1031 Fig. 6G).

1032 1033 Does MAMIP affect response bias in anger detection?

1034 MAMIP on anger detection performance were purely perceptual as those observed on the happiness 1035 detection task (in Experiments 1 and 2). Again, no significant decrement of fit was indeed found 
1036 when contrasting a full lme model including Congruency, Experiment and their interaction as fixed

1037 effects with a baseline lme model with no fixed effects at all ( $r_{c}$ slightly decreases from $0.97,95 \%$ CI

$1038[0.94,0.98]$ to $0.96,95 \%$ CI $\left.[0.92,0.97] ; \chi^{2}{ }_{3}=7.0, p=0.08\right)$. We confirmed this by post-hoc analyses

1039 showing that the response bias change due to congruency was statistically unreliable in both

1040 Experiment $3(-0.16,95 \%$ CI $[-0.32,0.01], t=-2.01, d f=9, p=0.075$; Fig. 6D) and $4(-0.038,95 \%$

1041 CI [-0.22, 0.14], $t=-0.48, d f=8, p=0.64$; Fig. $6 \mathrm{H}$ ).

\section{Symmetry of the action/emotion congruency effect}

1043 Our experiments univocally demonstrate that responses in our facial emotion detection task

1044 are, perceptually (not cognitively) determined by displayed emotion intensity, as well as by the

1045 congruency between bodily motor acts and emotion valence. The facilitation produced by emotional

1046 congruency, in addition of being independent of the arousing effect of goal-directed reaches (as

1047 demonstrated in Experiments 1 and 2), generalizes to the negative domain of affects (as

1048 demonstrated in Experiments 3 and 4) being thus also independent of action valence per se.

1049 Emotional congruency indeed facilitates the performance when the detection task is preceded by

1050 both a positive-comfortable action sequence (coupled with a positive emotion to be detected as in

1051 Experiments 1 and 2), and a negative-uncomfortable action sequence (coupled with a negative

1052 emotion to be detected as in Experiments 3 and 4).

1053 However, a major difference between our two sets of experiments is revealed by a strong 1054 emotion detection anisotropy consistent with an overall lower global sensitivity (average global d' of $10551.49,95 \%$ CI $[1.33,1.64]$ vs. $0.80,95 \%$ CI $[0.63,0.97]$ in Experiments $1 \& 2$ vs. 3 \& 4, respectively; 1056 Welch two sample $t=6.00, d f=75.11, p=0.00, d=1.36)$. This caused also a higher, though not 1057 significantly, threshold (average $A T$ was 6.32, 95\% CI [5.08, 7.56] vs. 8.49, 95\% CI [5.85, 11.12] in 1058 Experiments 1-2 vs. 3-4, respectively; Welch two sample $t=-1.51, d f=52.841, p=0.13, d=0.34$ )

1059 in the anger rather than in the happiness detection task. Such a bias was in line with results of the 1060 preliminary baseline experiment and with growing evidence in the emotion perception literature 1061 showing that realistic faces, as those used in the current experiments, often give rise to a happiness 1062 (rather than the more often found anger) detection advantage relative to both angry (Becker et al., 1063 2011; Becker et al., 2012; Juth et al., 2005) and sad (Srivastava \& Srinivasan, 2010) faces.

1064 In order to reconcile our data across emotions with different valence and to test (consistently 1065 with G3) the degree of symmetry of the facilitation-by-congruency effect, we recoded the four lme 1066 relationships describing the covariation of $d^{\prime}$ as a function of Morph Intensity and action/emotion 
1067 valence Congruency in our dataset in the generic emotion detection Cartesian space introduced in 1068 Fig. 2 (with $d^{\prime}$ for happiness detection on the $y$ axis and $d^{\prime}$ for anger detection on the $x$ axis). 1069 Consistently with an additive facilitation-by-congruency effect, the best fitting lme models resulting 1070 from the average $d^{\prime}$ by morph intensity relationships observed in Experiments $1 \& 2$ and Experiment 10712 \& 3 respectively, were both additively modulated by morph intensity and by congruency 1072 independently from action ordering. This gave rise to the following set of four lme regression lines:

1. a couple of regression lines for happiness detection with a common slope $\beta_{2 \text { happy }}$ of 1075

1076 about 0.27 and intercepts equal to -1.49 , for the action/emotion comfortable congruent condition $\left(\beta_{1 \text { happy comfortable }}\right)$, and -2.02 , for the action/emotion uncomfortable incongruent

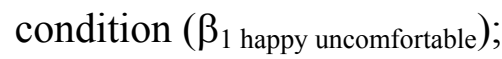

2. a couple of regression lines for anger detection with a common slope $\beta_{2}$ anger of about 0.181 and intercepts equal to -1.35 , for the action/emotion uncomfortable

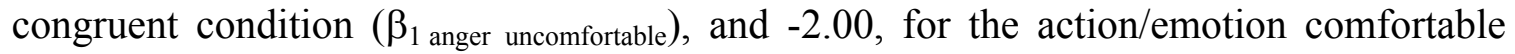

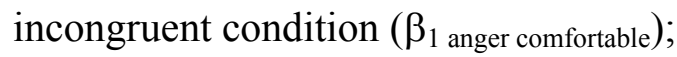

Entering the parameters of these four sets of lines into Equation 1 (pairing them appropriately

\section{3} following the procedure discussed in the Rationale and expectation section) allows recoding them into the emotion detection space. As shown in Fig. 7 this procedures defines two parallel lines with slope $\beta_{2 \mathrm{G}}=1.47$ : the blue solid line, standing for the average performance after comfortable reaches with $\beta_{1 \mathrm{G}}=1.465$, and the violet solid line, standing for the average performance after uncomfortable reaches with $\beta_{1 \mathrm{G}}=-0.025$. Importantly, the position of the grey dot representing the average $d^{\prime}$ in the baseline inaction condition relative to line of comfortable and uncomfortable reaches in the emotion detection space revealed an almost symmetric facilitation by congruency effect induced by MAMIP. The average $y$ coordinate of the baseline inaction point $(1.26 \pm 0.159)$, indeed, did not deviate significantly, along the happiness dimension, from the corresponding coordinate of the point along the line bisecting the space in between the comfortable and uncomfortable lines (0.935), standing for a perfectly symmetric effect (Welch two sample $t=1.4543$,

1094 coordinate of the point along the comfortable line (1.68), standing for an asymmetric effect fully in 1095 charge of the uncomfortable reaches (Welch two sample $t=1.98, d f=172, p=0.049, d=0.32$ ), and 1096 significantly larger than the corresponding coordinates of the point along the uncomfortable line 
1097 (0.19), standing for an asymmetric effect fully in charge of the comfortable reaches (Welch two 1098 sample $t=4.75, d f=172, p<0.001, d=0.72$ ).

1099

1100

1101

1102

1103

1104

1105

1106

1107

1108

1109

1110

1111

1112

1113

1114

1115

1116

1117

1118

1119

1120

1121

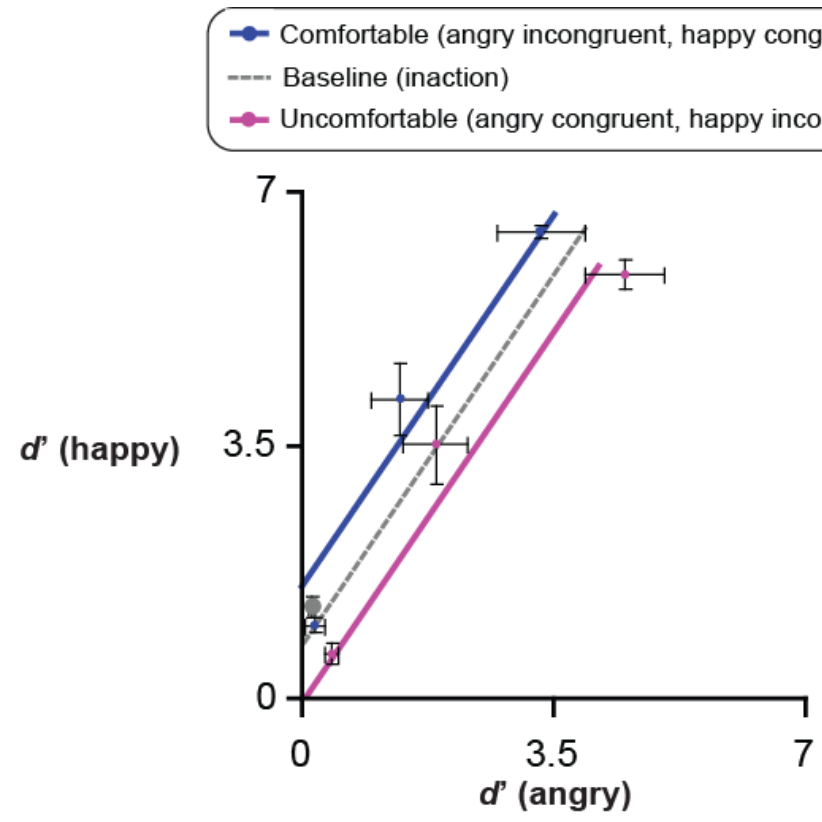

Figure 7. Comfortable and uncomfortable reaches impact sensitivity in an approximately symmetric way relative to a baseline inaction condition. The average $d^{\prime} \pm$ SEM in the baseline inaction condition (grey dot), is plotted in the emotion detection space together with average $d^{\prime} \pm$ SEM obtained in Experiments 1-4 (collapsed across Action Ordering conditions), with average performance to the happiness detection task on the $y$ axis, and average performance to the anger detection task on the $x$ axis. The two oblique solid lines represent the lme estimated $d^{\prime}$ calculated on the basis of individual $d^{\prime}$ after a sequence of comfortable (blue solid line) and uncomfortable reaches (violet solid line), according to Equation 1; with the blue line (line of comfortable reaches) representing estimated happy congruent (comfortable) $d^{\prime}$ as a function of estimated angry incongruent (comfortable) $d$ '; and the violet line (line of uncomfortable reaches) representing estimated happy incongruent (uncomfortable) $d^{\prime}$ as a function of estimated angry congruent (uncomfortable) $d^{\prime}$. The grey dotted line represents a reference over which the grey baseline dot is expected to lie on if the facilitation-by-congruency effect of action-induced mood on emotion sensitivity is perfectly.

The average coordinate of the baseline inaction point along the anger dimension $(0.15 \pm 0.049)$ instead deviates from the corresponding coordinates of all three lines: the line of perfect symmetry (Welch two sample $t$ vs. $0.37 \pm 0.049=3.20, d f=170, p=0.002, d=0.50$ ), the line of comfortable reaches (Welch two sample $t v s .-0.14 \pm 0.049=4.05, d f=170, p<0.001, d=0.62$ ), and the line of uncomfortable reaches (Welch two sample $t v s .0 .87 \pm 0.049=10.45, d f=170, p<0.001, d=1.60$ ).

Present results demonstrate that both comfortable and ucomfortable actions impact the perception of facial expression. However, while the facilitation-by-congruency effect on happiness detection is balanced across different types of actions, anger detection is facilitated by action induced mood congruency in a slight unbalanced way, with uncomfortable reaches being slightly more effective than comfortable reaches. 


\section{Conclusions}

1123 The present study demonstrates that the internal state of comfort/discomfort induced by

1124 reaching affects the detection of facial expressions in a direction consistent with the congruency

1125 between the valence of the action induced transient mood and the target emotion. Performance in a

1126 facial emotion detection task was indeed facilitated by congruent couplings between the valence of

1127 bodily actions performed before the task and emotions. This was revealed by a sensitivity

1128 enhancement and a consistent threshold decrement for facial expressions of emotion congruent with

1129 the valence of bodily motor acts, despite the absence of significant shifts in response bias: with

1130 happiness detection being facilitated by a sequence of comfortable reaches (Experiments 1 and 2),

1131 and vice versa with anger detection being instead facilitated by a sequence of uncomfortable

1132 reaches(Experiments 3 and 4).Importantly, these effects were consistent with an additive (not a

1133 multiplicative) facilitation-by-congruency effect, being the performance increment due to

1134 congruency almost constant at increasing morph intensities for both positive and negative target

1135 emotions. Notably, neither arousal by motor activation (predicting the opposite results in

1136 Experiments 1-2) nor action valence (comfort/discomfort of bodily action per se) can account for

1137 such effects. Furthermore, the systematic sensitivity changes produced by MAMIP did not cause

1138 analogous changes in response bias, demonstrating a full dissociation in our task between the way

1139 the internal states induced by action affect stimulus encoding (i.e., perception) vs. response selection

1140 (i.e., decision).

1141 We interpreted such a dissociation as a compelling evidence for a true top-down effect (in

1142 particular, action induced transient mood) on perception of tertiary qualities (in particular, perceived

1143 facial expressions): facial expression of emotion is penetrated by observer's internal states induced

1144 by bodily action, producing a facilitation by action/emotion valence congruency effect dependent on

1145 stimulus encoding rather than on response selection (Spruyt et al., 2002). This is consistent with an

1146 encoding stimulus account of affective priming indirectly produced by our bodily actions (i.e.,

1147 indirect affective priming, see Janiszewski \& Wyer, 2014). The reaching sequence performed before

1148 the detection task acted as an affectively polarized prime pre-activating the memory representations

1149 of affectively related facial features, thus making it easier to encode emotional facial features

1150 belonging to the same valence domain rather than to a different one.

1151 Notably, the way comfortable and uncomfortable reaches impacts the perception of facial

1152 expression in our study is almost symmetric relative to the baseline inaction condition. This is 
1153 consistent with the idea that, relative to the neutral mood experienced during inaction, the internal

1154 mood state of the actor is effectively modulated by both the musculoskeletal discomfort induced by

1155 reaching beyond a critical distance (where the arm is no longer sufficient) (Conway, 1999; Mark et

1156 al., 1997) and the empowerment of motor skillfulness induced by reaching within the peripersonal

1157 space. This sets the stage for the occurrence of a mood-congruency effect in emotion perception.

1158 Despite the consistency of our results, our study revealed a rather strong happiness detection

1159 advantage. This result casts our data on sensitivity-by-affect spaces with different metrics and thus 1160 statistically incomparable across emotions. In order to extract a general index (i.e., irrespective of 1161 emotion type) of the facilitation-by-congruency effect produced by MAMIP we thus mapped 1162 performance in the Cartesian space of action/emotion valence congruency (Fig. 8). In such a space 1163 each individual $d$ ' value relative to detection of an emotion congruent with reaching valence (on the $1164 y$ axis) is coupled with the corresponding $d$ ' value relative to detection of an emotion incongruent 1165 with reaching valence (on the $x$ axis). Representing each individual performance after a reaching 1166 session (either congruent or incongruent with the target emotion) as three points (one for each morph 1167 intensity) in the Cartesian space with the $d^{\prime}$ after the congruent condition in the $y$ axis and the $d^{\prime}$ after 1168 the incongruent condition in the $x$ axis thus provides a compact way to represent performance.

1169 The representation in Fig. 8 is indeed independent from absolute target emotion intensities and 1170 thus optimal for testing the facilitation-by-congruency effect in its general form. In the Cartesian 1171 space of emotion/action congruency an additive facilitation-by-congruency effect of MAMIP would 1172 be characterized by a family of lines with unitary slope and positive intercept: the value of the 1173 intercept being proportional to the sensitivity gain produced by action/emotion valence congruency 1174 (i.e., $d^{\prime}$ after congruent - $d^{\prime}$ after incongruent), independent of the emotion to be detected. A 1175 multiplicative facilitation-by-congruency effect would instead be characterized by a family of lines 1176 with null intercept and positive slope: the value of the slope being proportional to the relative gain 1177 produced by action/emotion valence congruency (i.e., ratio between $d^{\prime}$ after congruent and $d^{\prime}$ after 1178 incongruent).

1179 The co-variation found in our data between $d^{\prime}$ after congruent and incongruent reaches across 1180 types of target emotion, morph intensities and actions ordering conditions was clearly consistent with 1181 a model of the MAMIP effectiveness based on an additive (not multiplicative) facilitation-by1182 congruency hypothesis. This is shown in Fig. 8, where we recoded our 24 average $d^{\prime}$ into 12 points 1183 distributed linearly along the Cartesian space of emotion/action congruency. This allowed us to 
1184 predict individual detection performance after the action/emotion valence congruent condition ( $y$

1185

1186

1187

1188

1189

1190

1191

1192

1193

1194

1195

1196

1197

1198

1199

1200

1201

1202

1203

1204

1205

1206 axis) by means of the individual detection performance after the action/emotion valence incongruent condition ( $x$ axis) through lme regression. In both action ordering conditions, the 6 pairs of points (6 circles for uncomfortable $\Rightarrow$ comfortable; 6 triangles for comfortable $\Rightarrow$ uncomfortable) representing the average joined performance with positive and negative emotions are all placed in the positive half of the Cartesian space of emotion/action congruency and are well aligned along regression lines, fitting individual performances $(\mathrm{N}=117)$ with unitary slope (uncomfortable $\Rightarrow$ comfortable, cyan line: $1.0135 \pm 0.0237 ; t=0.760, d f=83.83, p=0.45$; comfortable $\Rightarrow$ uncomfortable, red line: $0.96 \pm 0.018 ; t=-1.90, d f=83.86, p=0.07)$ and similar positive intercept (uncomfortable $\Rightarrow$ comfortable, cyan line: $0.35 \pm 0.11 ; t=3.70, d f=83.83, p=0.00$; comfortable $\Rightarrow$ uncomfortable, red line: $0.34 \pm 0.10 ; t=4.306, d f=83.86, p=0.00$ ).

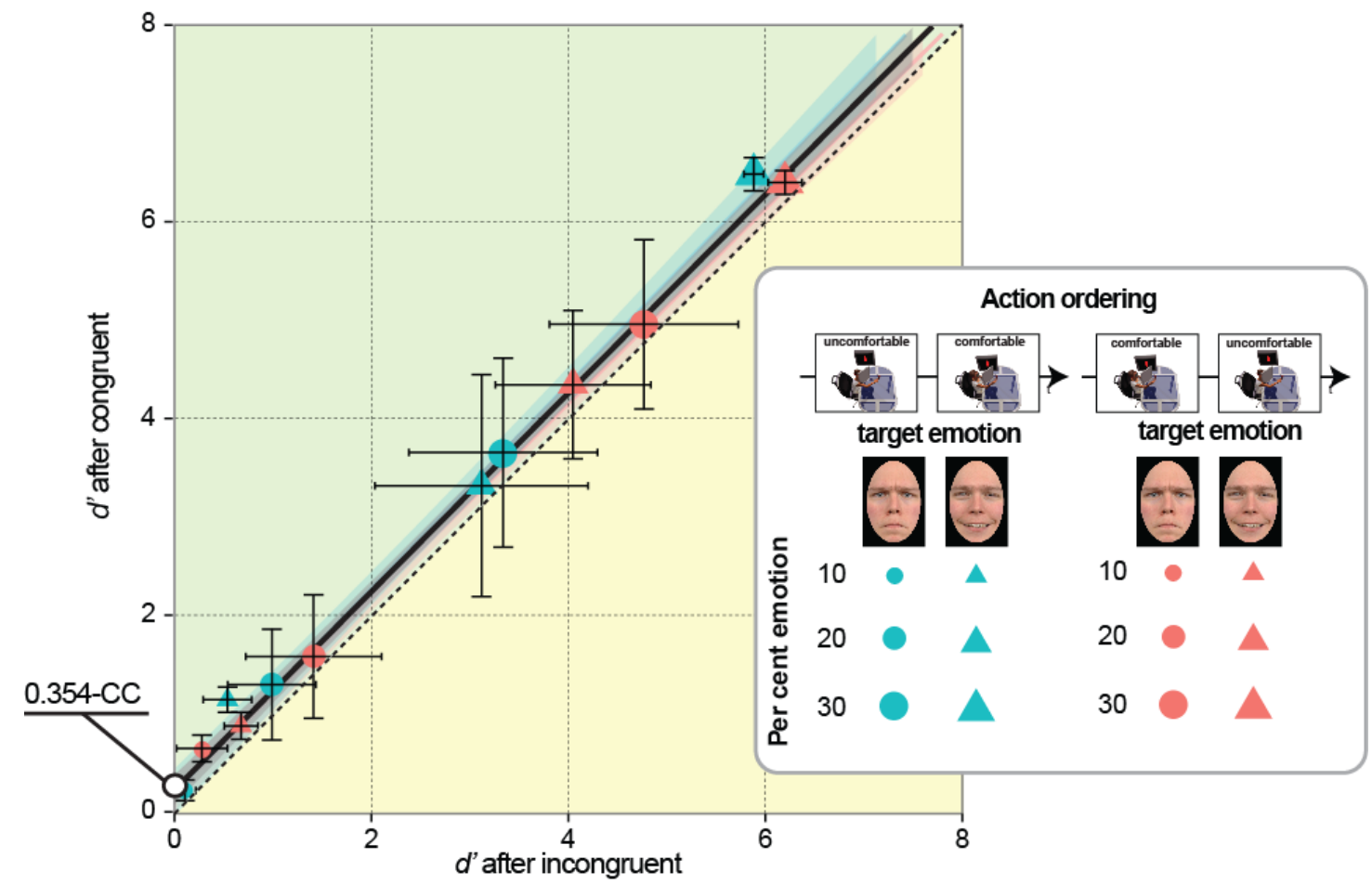

Figure 8. Joining emotion detection performances in the Cartesian space of emotion/action congruency. Average $d$ 'values resulting from each unique combination of morph intensity, target emotion, and action ordering after the congruent condition (on the $y$ axis) as a function of corresponding average $d^{\prime}$ values after the incongruent condition (on the $x$-axis). Vertical and horizontal error bars represent \pm SEM after congruent and incongruent reaches respectively. A point lying in the green half of such a Cartesian space represents a detection performance facilitated by congruency. The black dashed line cutting the Cartesian space in two equal halves is the reference for evaluating the overall additivity of the effect revealed by all four experiments: only points arranged along a line parallel to such a reference indeed denote an additive effect. In the legend: action ordering is coded by color (cyan for uncomfortable $\Rightarrow$ comfortable; red for comfortable $\Rightarrow$ uncomfortable); morph intensity by element size (small, medium, large corresponding to $10,20,30 \%$ morph respectively); the two target emotions by shape (circles for anger; triangles for happiness). The red, cyan and grey lines are the lme model regression lines (cyan fitting the uncomfortable $\Rightarrow$ comfortable performances; red fitting the 
1207 comfortable $\Rightarrow$ uncomfortable performances; grey fitting the entire data set regardless of the action ordering) and the 1208 shaded region corresponds to \pm standard error of the regression. The best fitting lme includes the $d$ ' after incongruent 1209 reaches as the only predictor and is characterized by a lme regression line (the grey line) with unitary slope and positive

12100.345 intercept evidenced at the margin of the graph.

1211

1212

This is confirmed by the results of the lme analysis testing the effects of the type of emotion,

1213 action orderings and morph intensity on individual $d^{\prime}$ after congruent reaches, once the effect of

1214 individual $d^{\prime}$ after incongruent reaches is controlled. $\left(t=85.41, d f=102, p<0.001 ; r^{2}=0.986,95 \%\right.$

1215 CI [0.978, 0.99], $r c=0.99,95 \%$ CI [0.989, 0.995]). In this model the likelihood of sensitivities to the

1216 detection of (general) emotion after congruent reaches was explained only by sensitivities to the

1217 detection of (general) emotion after incongruent reaches $\left(\beta=0.74 \pm 0.15, F_{1,82.85}=88.511, p<\right.$

1218 0.001). Furthermore, no significance decrement of fit was found when contrasting this model with a

1219 model (Fig. 8, grey regression line) with $d^{\prime}$ after incongruent reaches as the only covariate $(t=86.1$,

$1220 d f=102, \mathrm{p}<0.001$; both $r^{2}$ and $r_{c}$ remained unvaried to $0.986,0.99,95 \%$ CI [0.989, 0.995]

1221 respectively; $\left.\chi^{2}{ }_{2}=0.34, p=0.84\right)$. This model resulted to have both a unitary slope $(0.986 \pm 0.015 ; t$

$1222=-0.702, d f=167.9, p=0.484)$ and a positive intercept of about $0.345 \pm 0.075$ generic $d^{\prime}$ units $(t=$

$12235.308, d f=167.6, p<0.001)$.

1224 In our study, therefore, there is no evidence that morph intensity, actions ordering and type of 1225 emotion per se contributes to the determination of $d^{\prime}$ after congruent reaches beyond what $d^{\prime}$ after 1226 incongruent reaches can explain. Furthermore results were strikingly consistent with the predictions 1227 rising from a general additive facilitation-by-congruency effect of MAMIP. According to our 1228 Cartesian space of emotion/action congruency indeed: (1) the unitary lme estimated slope 1229 demonstrates the additive effect of MAMIP producing constant $d$ ' increments in congruent over 1230 incongruent conditions at increasing per cent emotion in the morph (regardless of target emotion); 1231 (2) the positive intercept, being equal to 0.345 , denotes the constant gain in the facial emotion 1232 detection performance produced by a congruency between the valence of the action preceding the 1233 detection task and the target emotion.

1234 Notably, the 0.345 Congruency Constant $(0.345-\mathrm{CC})$ is expressed in a generic $d^{\prime}$ scale 1235 independent of the valence of the emotion to be detected, being it representative of the action 1236 induced congruency advantage for both positive and negative facial expression of emotions. The 1237 0.345-CC thus quantifies the generic gain induced by bodily comfort over discomfort associated to 1238 motor actions on the detection of subtle variations in facial expressions of emotions with positive 1239 over negative valence (and vice versa), occurring in the absence of significant shifts in response bias. 
1240 The $0.345-\mathrm{CC}$ is thus conceivable as the first general constant of how bodily actions regulate 1241 affective perception.

1242 Our additive facilitation-by-congruency effect thus has several close relatives with accounts 1243 suggesting that different kinds of memory processes (from implicit to explicit) provide a strong 1244 linkage between the perceptual representation of a scene, the action plan representation, and the 1245 motor simulation (Barsalou, 2003; Nummenmaa et al., 2014). These accounts are indeed all related 1246 to the body of literature suggesting that the visual perception of objects and/or context can prime 1247 compatible/congruent actions/representations. As a case point, Tucker and Ellis (1998) found faster 1248 left- and right-hand responses when the agent was asked to make a decision about an object that 1249 could be grasped with the left and right hands, respectively (but see also, Tipper, Howard \& Jackson, 1250 1997; Glover, Rosenbaum, Graham \& Dixon, 2004). Similarly motor planning and execution has 1251 been found to be affected by different other aspects of the context like social intention (Becchio et al, 1252 2008a; Becchio et al, 2008b; Sartori et al 2009; Ferri et al., 2011; Quesque et al., 2013), social status 1253 (Fantoni et al., under review), end-goal accuracy (Ansuini, Santello, Massaccesi, Castiello, 2006), 1254 and motor affordances determined on the basis of biomechanical compatibility, relative to size, 1255 shape, and material properties of the object-hand system (Mon-Williams, Bingham, 2011; Flatters et 1256 al., 2012; Holt et al., 2013). In all these studies, aspects of the context make more accessible the 1257 memory specific features of actions that according to the influential planning-control model of 1258 actions (Glover, 2004) inform the planning component of prehensile movement. Following on the 1259 present results, we conjecture that the processing of tertiary qualities might be informed by a 1260 mechanism similar to the one activated by the viewing of a "right-hand" feature that primes a 1261 congruent right-handed motor response.

1262 Our findings shed light on the current debate voiced by Firestone \& Scholl (2015), between a 1263 more traditional "modular" view of perception, according to which visual processing is encapsulated 1264 from higher-level cognition (Fodor, 1983), vs. a tidal view of perception, according to which visual 1265 processing do instead access to more information elsewhere in the mind than has traditionally been 1266 imagined (Goldstone \& Barsalou, 1998). Specifically, our evidence in favor of a (limited) 1267 penetrability of perception challenges the bold claim that "cognition does not affect perception" 1268 (Firestone \& Scholl, 2015; main title), and supports the idea that observer's states linked to the 1269 valence of performed bodily acts might act as indirect affective primes, modulating stimulus 1270 encoding rather than response selection (Spruyt et al., 2002): bodily actions might prime contents 
1271 that have favorable or unfavorable motor implications and activate general evaluative concepts (e.g., 1272 positive vs. negative) thus affecting object properties experienced as external (i.e., perceptual in the 1273 phenomenological sense) and yet loaded with meaning.

1274 Our effect is theoretically relevant for the field of perceptual and cognitive sciences, although 1275 the existence of effects of observer's states on tertiary qualities should not look revolutionary 1276 (Firestone \& Scholl, 2015; section 4.2). Tertiary qualities - as defined in the Gestalt literature 1277 (Köhler, 1938; Metzger, 1941; Sinico, 2015; Toccafondi, 2009) - normally imply a reference to the 1278 observer, as reflected in the naïve psychology idea captured by "Beauty is in the eye of the 1279 beholder". Nevertheless, they are phenomenally objective (i.e., perceived as belonging to the object;

1280 Köhler, 1929) and show a remarkable - though not exclusive - dependence on configural stimulus 1281 properties. Therefore, assessing the extent and direction of observer-dependent effects on tertiary (in 1282 particular, expressive) qualities represents an important contribution to perceptual science, which 1283 can/must tolerate - we believe - some circumscribed leakage of cognition into perceptual 1284 apartments, consistent with grounded cognition (Barsalou, 2010; Kiefer \& Barsalou, 2013) among 1285 other perspectives.

1286 Finally, the present study complements the study by Fantoni and Gerbino (2014) and provides

1287 further evidence that mood congruency mediates the effects of motor action on perceived facial 1288 emotions, further showing the potential of MAMIP as an innovative and effective tool for the 1289 investigation of embodied cognition.

\section{Acknowledgements}

1291 We thank Matteo Manzini for helping with data collection.

\section{References}

1294 Ansuini C, Cavallo A, Bertone C, Becchio C. 2014. The visible face of intention: why kinematics matters. Front Psychol. July [cited 2015 Sept 17]; 5 [about 6pp.]. Available from: http://dx.doi.org/10.3389/fpsyg.2014.00815

1297 Barsalou LW. 2003. Situated simulation in the human conceptual system. Language and Cognitive 1298 Processes 18: 513-562.

1299 Barsalou LW. 2010. Grounded cognition: Past, present, and future. Topics in Cognitive Science 2: 716-724. 
1301 Bastiaansen JA, Thioux M, \& Keysers C. 2009. Evidence for mirror systems in emotions. Philosophical Transactions of the Royal Society B: Biological Sciences 364: 2391-2404.

1303 Bates D, Mechler M. 2014. Linear mixed-effects models using Eigen and S4. Cran.R project 1304 website. Available at http://cran.r-project.org/web/packages/lme4/lme4.pdf (accessed 2014 1305 September 2).

1306 Bates D. 2010. Ime4: Mixed-Effects Modeling with R. New York: Springer.

1307 Becchio C, Sartori L, Bulgheroni M, Castiello U. 2008a. Both your intention and mine are reflected in the kinematics of my reach-to-grasp movement. Cognition: 894-912.

Becchio C, Sartori L, Bulgheroni M, Castiello U. 2008b. The case of Dr. Jekyll and Mr. Hyde: a kinematic study on social intention. Consciousness and Cognition 17: 557-564.

Becker DV, Anderson US, Mortensen CR, Neufeld S, Neel R. 2011. The face in the crowd effect unconfounded: Happy faces, not angry faces, are more efficiently detected in the visual search task. Journal of Experimental Psychology: General 140: 637-59.

Becker DV, Neel R, Srinivasan N, Neufeld S, Kumar D, Fouse S. 2012. The vividness of happiness in dynamic facial displays of emotion. PLOS ONE 7: e26551. doi:10.1371/journal.pone.0026551 $257-262$.

Bouhuys AL, Bloem GM, Groothuis TG. 1995. Induction of depressed and elated mood by music influences the perception of facial emotional expressions in healthy subjects. Journal of Affective Disorders 33: 215-225.

Briscoe RE. 2014. Do intentions for action penetrate visual experience?. Frontiers in Psychology 5: 1265. doi: 10.3389/fpsyg.2014.01265

Cacioppo JT, Priester JR, Berntson GG. 1993. Rudimentary determinants of attitudes: II. Arm flexion and extension have differential effects on attitudes. Journal of Personality and Social Psychology 65: 5-17.

Cahour B. 2008. Discomfort, affects and coping strategies in driving activity. In Proceedings of the 15th European conference on Cognitive ergonomics: the ergonomics of cool interaction. ACM, 1-22. https://hal.archives-ouvertes.fr/hal-00671729.

Conway FT. 1999. Psychological mood state, psychosocial aspects of work, and musculoskeletal discomfort in intensive Video Display Terminal (VDT) work. International Journal of HumanComputer Interaction 11: 95-107. 
1332 Craighero L, Leo I, Umiltà C, Simion F. 2011. Newborns' preference for goal-directed actions. 1333 Cognition 120: 26-32.

1334 Derryberry D, Reed MA (1998) Anxiety and attentional focusing: Trait, state and hemispheric 1335 influences. Personality and Individual Differences 25: 745-761.

1336 Fantoni C, Gerbino W. 2014. Body Actions Change the Appearance of Facial Expressions. PLoS ONE 9: e108211. DOI:10.1371/journal.pone.0108211.

Fantoni C, Cavallero C, Gerbino W. 2014. The Motor Action Mood Induction Procedure affects the detection of facial emotions. In: Bernardis P, Fantoni C, Gerbino W, eds. TSPC2014 Proceedings of the Trieste Symposium on Perception and Cognition. Trieste: EUT, 79-81. http://www.openstarts.units.it/dspace/handle/10077/10499.

Fantoni C, Rigutti S, Piccoli V, Carnaghi A. under review. High social status attendees make prehensile movements faster and more accurate. PLoS ONE

1344 Fantoni C, Gerbino W, Kellman PJ. 2008. Approximation, torsion, and amodally-completed surfaces. Vision research 48: 1196-1216.

Farroni T, Csibra G, Simion F, Johnson M. 2002. Eye contact detection in humans from birth. Proceedings of the National Academy of Sciences, USA 99: 9602-9605.

Ferri F, Campione GC, Dalla Volta R, Gianelli C, Gentilucci M. 2011. Social requests and social affordances: how they affect the kinematics of motor sequences during interactions between conspecifics. PLOS ONE 6: e15855

Firestone C, Scholl B. 2015. Cognition does not affect perception: Evaluating the evidence for "topdown" effects. Behavioral and Brain sciences, 1-72.

Flatters IJ, Otten L, Witvliet A, Henson B, Holt RJ, Culmer P,et al. 2012. Predicting the effect of surface texture on the qualitative form of prehension. Plos One. Mar [cited 2015 Sept 17]; 7 [about 6pp.].

Available

from: http://journals.plos.org/plosone/article?id=10.1371/journal.pone.0032770 Fodor JA. 1983. The modularity of mind: An essay in faculty psychology. Cambridge: MIT Press.

Gasper K, Clore GL. 2002. Attending to the big picture: Mood and global versus local Processing of Visual Information. Psychological Science 13: 34-40.

1360 Gerbino W, Fantoni C, Nicolini C, Volcic R, Domini F. 2014. Active Multisensory Perception tool: BUS experience and action comfort. In: De Waard D, Brookhuis K, Wiczorek R, Di Nocera F, 
1362 Barham P, Weikert C, Kluge A, Gerbino W, Toffetti A, eds. Proceedings of the Human Factors and Ergonomics Society Europe. Groningen: hfse-europe, 403-413.

1364 Gerbino,W., Manzini, M., Rigutti S., \& Fantoni C. (2014) Action molds the perception of facial 1365 expressions. Perception 43: 173.

1366 Glover S. 2004. Separate visual representations in the planning and control of action. Behavioural and Brain Sciences 27: 3-24, discussion 24-78.

1368 Glover S, Rosenbaum DA, Graham J, Dixon P. 2004. Grasping the meaning of words. Experimental Brain Research 154: 103-108.

Goldstone RL, Barsalou LW. 1998. Reuniting perception and conception. Cognition 65: 231-262.

1371 Gratton G, Coles MGH., Sirevaag E, Eriksen CW, Donchin E. 1988. Pre- and post-stimulus activation of response channels: A psychophysiological analysis. Journal of Experimental Psychology: Human Perception and Performance 9: 793-810.

1374 Grossmann T, Cross ES, Ticini LF, Daum MM. 2013. Action observation in the infant brain: the role of body form and motion. Social neuroscience 8: 22-30.

Holt RJ, Lefevre AS, Flatters IJ, Culmer P, Wilkie RM, Henson BW, et al. 2013. Grasping the changes seen in older adults when reaching for objects of varied texture. Plos One. Jul [cited 2015 Sept 17]; $8 \quad$ [about 7 pp.]. Available from: http://journals.plos.org/plosone/article?id=10.1371/journal.pone.0069040

James W. 1884. What is an emotion? Mind 9: 188-205.

Janiszewski C, Wyer RS. 2014. Content and process priming: A review. Journal of Consumer Psychology 24: 96-118

Jeffries LM, Smilek D, Eich E, Enns JT. 2008. Emotional valence and arousal interact in the attentional blink. Psychological Science 19: 290-295.

Juth P, Lundqvist D, Karlsson A, Ohman A. 2005. Looking for foes and friends: Perceptual and emotional factors when finding a face in the crowd. Emotion 5: 379-395.

Kiefer M, Barsalou LW. 2013. Grounding the human conceptual system in perception, action, and internal states. In: Prinz W, Beisert M, Herwig A, eds. Action science: Foundations of an emerging discipline. Cambridge: MIT Press, 381-407.

1390 Kirsch W, Kunde W. 2013. Visual near space is scaled to parameters of current action plans. Journal of Experimental Psychology: Human Perception and Performance 39: 1313-1325. 
1392 Klauer KC, Musch J. 2003. Affective priming: Findings and theories. In: Musch J, Klauer KC., eds.

1393

1394

1395

1396

1397

1398

1399

1400

1401

1402

1403

1404

1405

1406

1407

1408

1409

1410

1411

1412

1413

1414

1415

1416

1417

1418

1419

1420

1421

1422

The psychology of evaluation: Affective processes in cognition and emotion. Mahwah, NJ: LEA, 7-49.

Kliegl R, Wei P, Dambacher M, Yan M, Zhou, X. 2010. Experimental effects and individual differences in linear mixed models: Estimating the relationship between spatial, object, and attraction effects in visual attention. Frontiers in Psychology 1: 238. DOI: 10.3389/fpsyg.2010.00238

Knoblauch K, Maloney LT. 2012. Modeling Psychophysical Data in R. New York: Springer.

Köhler W. 1929. Gestalt psychology. New York: Liveright.

Köhler, W. (1938). The place of value in a world of facts. New York: Liveright.

Langner O, Dotsch R, Bijlstra G, Wigboldus DHJ, Hawk ST, van Knippenberg A. 2010. Presentation and validation of the Radboud Faces Database. Cognition \& Emotion 24: 1377-1388.

Lebrecht S, Bar M, Barrett LF, Tarr MJ. 2012. Micro-valences: Perceiving affective valence in everyday objects. Frontiers in Psychology 3: 107. DOI: 10.3389/fpsyg.2012.00107

Lee DH, Susskind JM, Anderson AK. 2013. Social transmission of the sensory benefits of eye widening in fear expressions. Psychological Science 24: 957-965.

Leppänen JM, Nelson CA. 2009. Tuning the developing brain to social signals of emotions. Nature Reviews Neuroscience10: 37-47.

Macmillan NA, Creelman CD. 2004. Detection theory: A user's guide. Psychology press.

Mark LS, Nemeth K, Gardner D, Dainoff MJ, Paasche J, Duffy M, Grandt K. 1997. Postural dynamics and the preferred critical boundary for visually guided reaching. Journal of Experimental Psychology: Human Perception and Performance 23: 1365-1379.

Marneweck M, Loftus A, Hammond G. 2013. Psychophysical measures of sensitivity to facial expression of emotion. Frontiers in Psychology 4:63. DOI: 10.3389/fpsyg.2013.00063

Masson ME, Bub DN, Breuer AT. 2011. Priming of reach and grasp actions by handled objects. Journal of Experimental Psychology: Human Perception and Performance 37: 1470.

Metzger, W. (1941) Psychologie. Darmstadt: Steinkopff.

Mon-Williams M, Bingham GP. 2011. Discovering affordances that determine the spatial structure of reach-to-grasp movements. Experimental Brain Research 211: 145-160.

Nicolini C, Fantoni C, Mancuso G, Volcic R, Domini F. 2014. A framework for the study of vision in active observers. In: Rogowitz BE, Thrasyvoulos NP, Huib R, eds. Proc. SPIE 9014, Human 
1423

1424

1425

1426

1427

1428

1429

1430

1431

1432

1433

1434

1435

1436

1437

1438

1439

1440

1441

1442

1443

1444

1445

1446

1447

1448

1449

1450

1451

1452

Vision and Electronic Imaging XIX. Bellingham: International Society for Optics and Photonics, 1-14. DOI: $10.1117 / 12.2045459$.

Niedenthal PM, Halberstadt JB, Innes-Ker AH. 1999. Emotional response categorization. Psychological Review 106: 337-361.

Nummenmaa L, Glerean E, Hari R, Hietanen JK. 2014. Bodily maps of emotions. Proceedings of the National Academy of Sciences of the United States of America 111: 646-651.

Phelps EA, Ling S, Carrasco M. 2006. Emotion facilitates perception and potentiates the perceptual benefits of attention. Psychological Science 17: 292-299.

Pollak SD, Kistler DJ. 2002. Early experience is associated with the development of categorical representations for facial expressions of emotion. Proceedings of the National Academy of Sciences of the United States of America 99: 9072- 9076.

Quesque F, Lewkowicz D, Delevoye-Turrell YN, Coello Y. 2013. Effects of social intention on movement kinematics in cooperative actions. Frontiers in Neurorobotics 7:14. DOI: 10.3389/fnbot.2013.00014

Rigutti S, Fantoni C, Gerbino W. 2015. Web party effect: a cocktail party effect in the web environment. PeerJ 3:e828. https://dx.doi.org/10.7717/peerj.828

Santos LR, Hood BM. 2009. Object representation as a central issue in cognitive science. In: Hood, BM, Santos LR, eds. The Origins of Object Knowledge. Oxford: Oxford University Press, 2-24.

Sartori L, Becchio C, Bara BG, Castiello U. 2009. Does the intention to communicate affect action kinematics?. Consciousness and Cognition 8: 766-772.

Sinico M. 2015. Tertiary qualities, from Galileo to Gestalt psychology. History of the Human Sciences 28: 68-79. DOI:10.1177/0952695115591409

Sloan DM. 2004. Emotion regulation in action: emotional reactivity in experiential avoidance. Behaviour Research \& Therapy 42: 1257-1270.

Spruyt A, Hermans D, Houwer JD, Eelen P. 2002. On the nature of the affective priming effect: Affective priming of naming responses. Social Cognition 20: 227-256.

Srivastava P, Srinivasan N. 2010. Time course of visual attention with emotional faces. Attention, Perception, \& Psychophysics 72: 369-377.

Straulino E, Scaravilli T, Castiello U. 2015. Social intentions in Parkinson's disease patients: A kinematic study. Cortex 70: 179-188. 
1453 Tipper SP, Howard LA, Jackson SR. 1997. Selective reaching to grasp: Evidence for distractor 1454 interference effects. Visual Cognition 4: 1-38.

1455 Toccafondi, F. (2009). Facts, values, emotions, and perception. In: B. Centi \& W. Huemer eds. 1456 Values and ontology: Problems and perspectives. Frankfurt: Ontos Verlag.

1457 Tucker M, Ellis R. 1998. On the relations between seen objects and components of potential actions. 1458 Journal of Experimental Psychology: Human Perception and Performance 24: 830-846

1459 Vishton PM, Stephens NJ, Nelson LA, Morra SE, Brunick KL, Stevens JA. 2007. Planning to reach 1460 for an object changes how the reacher perceives it. Psychological Science 18, 713-719. doi: 10.1111/j.1467-9280.2007.01965.x

1462 Volcic R, Fantoni C, Caudek C, Assad J., Domini F. 2013. Visuomotor adaptation changes stereoscopic depth perception and tactile discrimination. Journal of Neuroscience 33: 1708117088.

1465 Vonesh EF, Chinchilli VM, Pu K. 1996. Goodness-of-fit in generalized nonlinear mixed-effects 1466 models. Biometrics 52: 572-587.

1467 Wilson TD, Gilbert DT. 2008. Explaining away a model of affective adaptation. Perspective in $1468 \quad$ Psychological Sciences 3: 370-386.

1469 Wickens CD. 1984. Processing resources in attention. In: Parasuraman R, Davis R, eds. Varieties of attention. New York: Academic Press.

Witt JK. 2011. Action's effect on perception. Current Directions in Psychological Science, 20: 201206.

Witt JK, Taylor JET, Sugovic M, Wixted JT. 2015. Signal detection measures cannot distinguish perceptual biases from response biases. Perception, 44: 289-300.

Woods AJ, Mennemeier M, Garcia-Rill E, Huitt T, Chelette KC, McCullough G, Munn T, Brown G, Kiser TS. 2012. Improvement in arousal, visual neglect, and perception of stimulus intensity following cold pressor stimulation. Neurocase 18: 115-122.

Woods AJ, Philbeck JW, Wirtz P. 2013. Hyperarousal decreases human visual thresholds. PLoS ONE 8: e61415. DOI:10.1371/journal.pone.0061415.

Yap AJ, Wazlawek AS, Lucas BJ, Cuddy AJC, Carney DR. 2013. The ergonomics of dishonesty: Science 24: 2281-2289. 
1483 Zadra JR, Clore GL. 2011. Emotion and perception: The role of affective information. Wiley 1484 Interdisciplinary Reviews: Cognitive Science 2: 676-685.

1485 Zeimbekis J, Raftopoulos A. 2015. The Cognitive Penetrability of Perception. Cambridge: MIT 1486 Press.

\section{Supplemental files}

1489 Supplemental_Dataset_S1. Data from the four experiments. Three worksheets are included in the

1490 file: (1) RAW_DATASET, with the set of 64 yes/no responses characterizing each individual raw

1491 performance; (2) $\mathrm{glm}$ values, with individual $d$ ' triplets associated to the three combinations of [N]

1492 and $[\mathrm{S}+\mathrm{N}]$ trials $(0-10,0-20,0-30 \%$ emotion in the morph), for congruent and incongruent

1493 conditions; (3) est. Grouped indices, with individual global d', $A T$, and $c$ values.

1494 Supplemental_Permission Letter. Letter of permission signed by a representative of Radboud

1495 database allowing PeerJ to publish pictures of model 28 used in the manuscript.

1496 Supplemental_Consent form. Consent form (Italian language) used in the Study with the approval

1497 of the Research Ethics Committee of the University of Trieste (approval number 52). 